\title{
Effects of Compressibility, Space Porosity, and Slip Flow on Peristaltic Transport of MHD Jeffrey Fluid through an Annulus: Aerospace Field Applications
}

\author{
Khaled S. Mekheimer ${ }^{\text {a, b }}$. Soliman R. Komy ${ }^{\text {c }}$. Sara I. Abdelsalam ${ }^{\text {d,* }}$ \\ a Mathematics \& Statistics Department, Faculty of Science, Taif University, Hawia (888) Taif, \\ Saudi Arabia \\ ${ }^{\mathrm{b}}$ Department of Mathematics, Faculty of Science(Men), Al-Azhar University, Nasr City, Cairo, \\ Egypt \\ ${ }^{\mathrm{c}}$ Department of Mathematics, Faculty of Science, Helwan University, Cairo, Egypt \\ d Basic Science Department, Faculty of Engineering, The British University in Egypt, Al-Shorouk \\ City, Misr-Suez Desert Road, P.O. Box 43, Cairo 11837, Egypt
}

\begin{abstract}
This work is concerned with peristaltic flow as a result of the influence of ultrasonic radiation on the flow of a liquid through the gap between two coaxial tubes (annulus) which deforms the wall of the outer tube in the shape of travelling transversal waves. The problem studies the effect of slip velocity and viscoelasticity on the dynamics of a compressible and electrically conducting Jeffrey fluid through a porous medium in the presence of a constant magnetic field. Navier-Stokes equations for the annulus are solved by means of a perturbation analysis, in which the ratio of the wave amplitude to the outer tube radius is a small parameter. In the second order approximation, the net flow induced by the travelling wave is calculated for various values of Reynolds number, relaxation time, retardation time, magnetic parameter, slip parameter, permeability, and annulus radius ratio. The calculations disclose that the compressibility, the radius ratio, and the non-Newtonian effects have a strong influence on the net flow rate induced. This problem has numerous applications in various branches of science, including stimulation of fluid flow in annuli under the effect of elastic waves, the production process of oil, and studies of blood flow dynamics in living creatures (catheter in an artery). The present study investigates novelties brought about into the classic peristaltic mechanism by inclusion of the annulus radius ratio and the non-Newtonian effects that were found to lessen the oscillatory behavior of the net flow rate. Several results of other models can be deduced as the limiting cases of our situation. Finally, variations of the emerging parameters embedded in the flow system are discussed numerically and graphically.
\end{abstract}

Keywords Peristaltic flow . Jeffrey fluid. Annulus . Slip effect . Porous medium .

Compressible flow

\section{Introduction}

It is accepted now that the majority of biological and industrial fluids are non-Newtonian because it is known that non-Newtonian effects produce unexpected changes in fluid behavior through viscoelastic regimes. Unlike the Newtonian fluids, the non-Newtonian fluids cannot be described by a single constitutive relationship between stress and strain rates. Such constitutive equations give rise to complicated mathematical problems and thus, the mathematicians, modelers, physicists, and computer scientists encounter wide variety of challenges in constructing analytical and numerical solutions. Ali et al. [2] have studied the peristaltic flow of a Maxwell fluid in a channel with compliant walls. Elkoumy et al. [9] have studied peristaltic transport through porous medium of a Maxwell model in the presence of magnetic field and Hall effect. Hayat et al. [13] studied peristaltic flow of a Maxwell fluid in a porous medium in the presence of Hall effects. The models in which beside the time derivatives of the stress tensor also the derivative of the rate of strain tensor is included show relaxation as well as retardation behavior. An important example of this kind is the Jeffrey's version of the Oldroyd model [5, 17, 20, $29,32]$. This fluid model includes elastic and memory effects exhibited by dilute polymer solutions and biological liquids. Industrial and biological flows in pipes are quite * Corresponding author:

E-mail address: $\underline{\text { sara.abdelsalam@bue.edu.eg, siabdelsalam@yahoo.com }}$ 
common and have relevant applications. Biofluids, in general, are propelled in ducts by means of transverse progressive waves propagating along the walls of the ducts. This process is called peristalsis. The flow of urine through the ureter, the movement of chyme in the entire gastro-intestinal tract, and the transport of the food grains and liquids from the mouth through the oesophagus are very common examples of this mechanism in the physiological world. Biomechanical pump works in accordance with the same mechanism are man-made instruments to pump physiological fluids. Several investigations on peristaltic transport through circular cylindrical tubes have been carried out by different researchers for Newtonian fluids $[3,34,36,37,41]$ as well as for non-Newtonian ones [6, $31,35]$.

The compressible flow is a subset of fluid mechanics/hydraulics, and therefore the knowledge development followed the understanding of the incompressible flows. Most of the investigations show that the compressibility has an interesting effect on liquid flow. Aarts and Oams [1] studied the influence of ultrasonic radiation on the flow of a liquid through a porous medium in an axisymmetric cylindrical pore, taking the liquid compressible. Tsiklauri and Bresenev [39] developed the study of Aarts and Ooms by taking the non-Newtonian properties of the fluid into account. Hayat et al. [15] took into consideration the retardation time for a Jeffrey fluid that resulted in oscillation decay for the net flow rate. Eldesoky [7] studied the peristaltic transport for a Maxwell fluid in the presence of a slip condition, while Eldesoky and Mousa [8] studied the case of compressible fluid in a porous medium. Also, Mekheimer and Abdel-Wahab [24] studied the same compressibility effects for a Maxwell fluid but in an annulus.

In the literature, much attention has been given to the flow problems with no-slip condition. This is not realistic because many polymeric fluids slip or stick-slip on solid boundaries, and hence the no-slip condition is no longer valid. The inadequacy of the noslip condition is quite evident in polymer melts which often exhibit microscopic wall slip. The fluids that exhibit boundary slip have important technological applications such as in polishing valves of artificial heart and internal cavities. Also, there is a disagreement in the dynamic response between experimental values and the theoretical prediction. One of the possible causes of this disagreement in the response amplitude is because the fluid was locally under higher shear rates than its Maxwellian limit. If the shear stress at the wall is greater than the critical shear stress, the flow slips at the wall and conversely if the shear stress is not large enough, then the classical Poisseuille solution with no-slip is observed. So, the slip seems to be critical in determining the characteristics of the flow [7]. The effects of slip boundary conditions on the compressible flow were discussed in details by El-Shehawy et al. [12].

In mathematics, an annulus (the Latin word for "little ring") is a ring-shaped geometric figure, or it is the area between two concentric circles. Studying the flow between coaxial tubes where the outer tube has a sinusoidal wave travelling down its wall and the inner one is rigid (annulus) has taken a great portion of scientific research [21]. The peristaltic flow through a porous medium in an annulus was studied in [22]. The couple stress fluid was studied in [23] with application in the endoscope. Catheter applications were studied in [26] for an incompressible Newtonian fluid. The Casson fluid was studied in an annulus [28], and [27] took it as an application to catheterized artery. Worthy to say that Catheterization refers to a procedure in which long, thin, flexible plastic tube (catheter) is inserted into an artery. Catheter procedures can both diagnose and treat heart and blood vessels condition. The insertion in an artery will form an annular region between the walls of the catheter and the artery and this alters the flow field [25].

Flow through a porous medium has been of considerable interest in recent years particularly among geophysical fluid dynamicists. Some progress has been made in the presence of porous media. For recent contributions, we refer to some interesting studies in the references $[9,11,18,30,33]$. The most general study of stimulation of fluid flow in porous media via peristaltic mechanism is presented in Ref. [1], which has been used as a starting point in order to include non-Newtonian effects into the peristaltic model.

The magnetohydrodynamic (MHD) flow of a fluid in a channel with elastic, rhythmically contracting walls (peristaltic flow) is of interest in connection with certain 
problems of the movement of conductive physiological fluids, e.g., blood. Peristaltic motion of a generalized Newtonian fluid under the effect of a transverse magnetic field is studied by El-Shehawey et al. [10]. Elkoumy et al. [9] studied the peristaltic transport of a Maxwell fluid through a porous medium. Controlling positions of catheters using magnetic fields has been investigated by [40], where a computerized closed-loop control system for navigating catheters and other interventional devices containing a permanent magnet near its distal tip have been used, and thus, an external magnetostatic field is applied to the vicinity of the tissue where the medical procedure is being performed in order to orient the catheter tip.

To the best of the author's knowledge, no attempt has yet been made to discuss the peristalsis of non-Newtonian Jeffrey fluid through a porous medium taking the compressibility of the liquid and the magnetic field into account. Therefore, the main purpose of this paper is devoted to observe the effects of the insertion of a catheter in an artery. The mathematical model considers the flow of a compressible viscous Jeffrey fluid between the annular space (gap) of two concentric tubes; the outer tube (circular cylinder) corresponds to the artery and the inner one (solid circular cylinder) to the catheter. The flow is induced by sinusoidal peristaltic waves along the length of the outer tube wall (artery wall). In this article, we follow Yin and Fung [13] by taking the compressibility of the liquid into account and the flow between two coaxial tubes. We extend the analysis of Mekheimer and Abdel-Wahab [24] to study the magnetohydrodynamic peristaltic motion of a viscous compressible Jeffrey fluid through a flexible porous annulus with boundary slip. We further extend the analysis of Tsiklauri and Bresenev [39] by taking the flow into an annulus.

We formulate the problem in Section 2. In Section 3, we solve the problem. The results and discussion as well as the conclusions are presented in Sections 4 and 5, respectively. We have found that oscillatory decay strongly depends on the annulus radius ratio as on the retardation time.

\section{Formulation of the problem}

We consider a viscous, compressible, and electrically conducting Jeffrey fluid in between flexible axisymmetric coaxial tubes. The inner tube is kept rigid and the flow in the porous space is due to sinusoidal wave travelling down the walls of the outer tube. We introduce the cylindrical coordinates $(\mathrm{r}, \theta, \mathrm{z})$ with the $\mathrm{z}$-axis along the centerline of the inner tube. The fluid is subjected to a constant transverse magnetic field $B_{o}$ where the induced magnetic field is assumed negligible. The geometry of the wall surface is described in Fig. 6.1. The equations for the radii are

$$
\mathrm{R}_{1}=a_{1}, \quad \mathrm{R}_{2}(\mathrm{z}, \mathrm{t})=a_{2}+\mathrm{b} \cos \frac{2 \pi}{\lambda}(z-\mathrm{c} t) .
$$

where $a_{1}, a_{2}$ are the radii of the inner and outer tubes respectively, $\mathrm{b}$ is the amplitude of the wave, $\lambda$ is the wavelength and $\mathrm{c}$ is the propagation velocity along $\mathrm{z}$-direction.

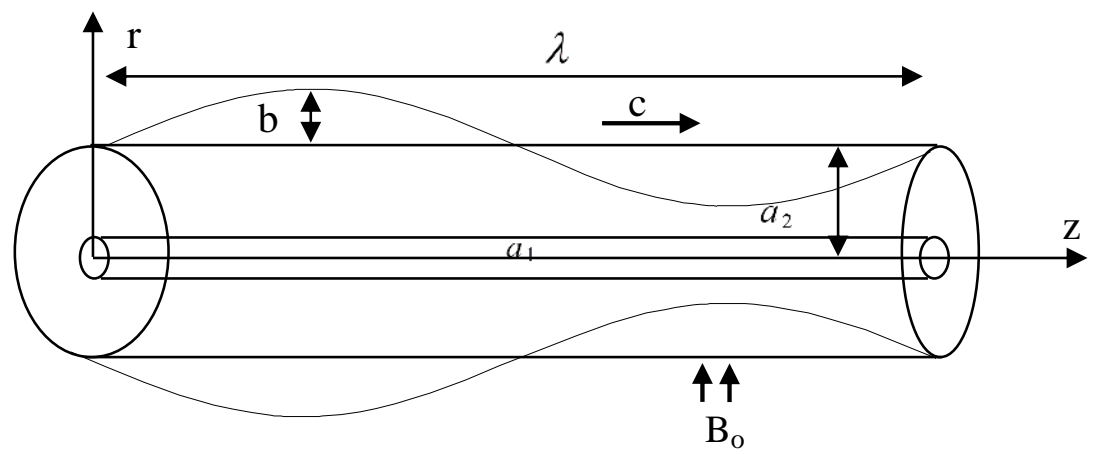

Fig. 1 Geometry of the problem 
The flow is governed by the mass and momentum laws given by

$$
\begin{gathered}
\frac{\partial \rho}{\partial t}+\nabla \cdot(\rho \mathbf{V})=0, \\
\rho\left[\frac{\partial}{\partial t}+(\mathbf{V} . \nabla)\right] \mathbf{V}=\operatorname{div} \mathbf{T}+\mathbf{R}+\mathbf{J} \times \mathbf{B},
\end{gathered}
$$

where $\rho$ and $t$ are the density of fluid and the time. Also, $\mathbf{V}, \mathbf{J}, \mathbf{R}$, and $\mathbf{B}$ are the velocity vector, electric current density, Darcy's resistance in the porous medium, and magnetic induction vector, respectively. The constitutive equations for a Jeffrey fluid are [15]

$$
\begin{gathered}
\mathbf{T}=-p \mathbf{I}+\mathbf{S}, \\
\left(1+\lambda_{1} \frac{\partial}{\partial t}\right) \mathbf{S}=\mu\left(1+\lambda_{2} \frac{\partial}{\partial t}\right) \dot{\gamma}, \\
\dot{\gamma}=\nabla \mathbf{V}+(\nabla \mathbf{V})^{\dagger}-\frac{2}{3} \nabla \cdot \mathbf{V},
\end{gathered}
$$

where $\mathbf{T}, \mathbf{S}$, and $\mathbf{I}$ are the Cauchy stress tensor, extra stress tensor, and the identity tensor, respectively. And $\mathrm{p}, \lambda_{1}, \lambda_{2}, \dagger, \mu$ are the pressure, the relaxation time, the retardation time, the transpose, and the dynamic viscosity, respectively.

On the basis of Jeffrey fluid model [38], the following expression of Darcy's resistance has been suggested:

$$
\left(1+\lambda_{1} \frac{\partial}{\partial t}\right) \mathbf{R}=-\frac{\mu \Phi}{\mathrm{K}}\left(1+\lambda_{2} \frac{\partial}{\partial t}\right) \mathbf{V},
$$

where $\Phi(0<\Phi<1)$ and K ( > 0) are, respectively, the (constant) porosity and permeability of the porous medium. The conducting fluid is permeated by an imposed uniform magnetic field $\mathrm{B}_{0}$ which acts in the transverse direction. For low magnetic Reynolds number, the induced magnetic field can be ignored, the magnetic body force $\mathbf{J} \times \mathbf{B}$ becomes $\sigma(\mathbf{V} \times \mathbf{B}) \times \mathbf{B}$ when imposed, induced electric fields are negligible, and only the magnetic field $\mathbf{B}$ contributes to the current $\mathbf{J}=\sigma(\mathbf{V} \times \mathbf{B})$. Here, $\sigma$ is the electrical conductivity of the fluid.

The characteristic response of the liquid to a compression is described by the constitutive equation

$$
\frac{1}{\rho} \frac{\partial \rho}{\partial t}=k_{c}
$$

where $k_{c}$ is the compressibility of the liquid. The solution to (8) for the density as a function of the pressure is given by

$$
\rho=\rho_{o} \exp \left(k_{c}\left(p-p_{c}\right)\right)
$$

where $\rho_{o}$ is the (constant) density at the reference pressure $p_{c}$. With the flow parameters independent of the azimuthal coordinate $\theta$, the velocity takes the form

$$
\mathbf{V}=\mathrm{u}(r, z, t) \underline{e}_{r}+\mathrm{w}(r, z, t) \underline{e}_{z},
$$

where $\underline{e}_{r}$ and $\underline{e}_{z}$ are unit vectors in the positive $\mathrm{r}$ and z direction, respectively.

In cylindrical coordinates the continuity equation (2) reads

$$
\frac{\partial \rho}{\partial t}+\mathrm{u} \frac{\partial \rho}{\partial r}+\mathrm{w} \frac{\partial \rho}{\partial z}+\rho\left(\frac{\partial \mathrm{u}}{\partial r}+\frac{\mathrm{u}}{r}+\frac{\partial \mathrm{w}}{\partial z}\right)=0
$$

Upon making use of Eqs. (5), (6), (7) into Eq. (3), the equation of momentum becomes

$$
\begin{aligned}
& \left(1+\lambda_{1} \frac{\partial}{\partial t}\right)\left[\rho \frac{\partial u}{\partial t}+\rho\left(\mathrm{u} \frac{\partial \mathrm{u}}{\partial r}+\mathrm{w} \frac{\partial \mathrm{u}}{\partial z}\right)\right]=-\left(1+\lambda_{1} \frac{\partial}{\partial t}\right) \frac{\partial p}{\partial r} \\
& +\mu\left(1+\lambda_{2} \frac{\partial}{\partial t}\right)\left[\frac{\partial^{2} \mathrm{u}}{\partial r^{2}}+\frac{1}{r} \frac{\partial \mathrm{u}}{\partial r}-\frac{\mathrm{u}}{r^{2}}+\frac{\partial^{2} \mathrm{u}}{\partial z^{2}}+\frac{1}{3} \frac{\partial}{\partial r}\left(\frac{\partial \mathrm{u}}{\partial r}+\frac{\mathrm{u}}{r}+\frac{\partial \mathrm{w}}{\partial z}\right)\right]
\end{aligned}
$$




$$
\begin{aligned}
& -\frac{\mu \Phi}{\mathrm{K}}\left(1+\lambda_{2} \frac{\partial}{\partial t}\right) \mathrm{u}-\sigma \mathrm{B}_{\mathrm{o}}^{2}\left(1+\lambda_{1} \frac{\partial}{\partial t}\right) \mathrm{u}, \\
& \left(1+\lambda_{1} \frac{\partial}{\partial t}\right)\left[\rho \frac{\partial \mathrm{w}}{\partial t}+\rho\left(\mathrm{u} \frac{\partial \mathrm{w}}{\partial r}+\mathrm{w} \frac{\partial \mathrm{w}}{\partial z}\right)\right]=-\left(1+\lambda_{1} \frac{\partial}{\partial t}\right) \frac{\partial p}{\partial z} \\
& +\mu\left(1+\lambda_{2} \frac{\partial}{\partial t}\right)\left[\frac{1}{r} \frac{\partial}{\partial r}\left(r \frac{\partial \mathrm{w}}{\partial r}\right)+\frac{\partial^{2} \mathrm{w}}{\partial z^{2}}+\frac{1}{3} \frac{\partial}{\partial z}\left(\frac{\partial \mathrm{u}}{\partial r}+\frac{\mathrm{u}}{r}+\frac{\partial \mathrm{w}}{\partial z}\right)\right] \\
& -\frac{\mu \Phi}{\mathrm{K}}\left(1+\lambda_{2} \frac{\partial}{\partial t}\right) \mathrm{w}-\sigma \mathrm{B}_{\mathrm{o}}^{2}\left(1+\lambda_{1} \frac{\partial}{\partial t}\right) \mathrm{w} .
\end{aligned}
$$

At the boundary $R_{2}$ the fluid is subjected to the motion of the outer wall with slip effect. For the slip flow the fluid still obeys Navier-Stokes equation, but the no-slip condition is replaced by the slip equation $\mathrm{u}_{\mathrm{t}}=\mathrm{A} \frac{\partial \mathrm{u}_{\mathrm{t}}}{\partial n}$ where $\mathrm{u}_{\mathrm{t}}$ is the tangential velocity, $n$ is the normal to the surface, and A is the coefficient close to mean free path of the molecules of the fluid. This condition has been attributed to Beavers and Joseph [4] for a porous boundary, but it was Navier who proposed it a century ago. Although Navier condition looked simple, analytically it is more difficult than the no-slip condition. The boundary conditions at the inner and outer walls thus requires

$$
\begin{gathered}
\mathrm{u}\left(\mathrm{R}_{1}, z, t\right)=0, \quad \mathrm{w}\left(\mathrm{R}_{1}, z, t\right)=0 . \\
\mathrm{u}\left(\mathrm{R}_{2}, z, t\right)=\frac{\partial \mathrm{R}_{2}}{\partial y}, \quad \mathrm{w}\left(\mathrm{R}_{1}, z, t\right)=\mathrm{A} \frac{\partial \mathrm{w}}{\partial \mathrm{r}} .
\end{gathered}
$$

Finally, the flow rate $\mathrm{Q}$ through the tube is given by

$$
<\mathrm{Q}>=2 \pi \int_{\mathrm{R}_{1}}^{\mathrm{R}_{2}} \mathrm{w}(r, z, t) r d r .
$$

The equations are made dimensionless by scaling length by $a_{2}$ and time by $a_{2} / \mathrm{c}$. Furthermore, we introduce the dimensionless variables

$$
\begin{aligned}
& \tilde{\mathrm{R}}_{1}=\mathrm{R}_{1} / a_{2}, \tilde{\mathrm{R}}_{2}=\mathrm{R}_{2} / a_{2}, \tilde{\rho}=\rho / \rho_{o}, \tilde{\mathrm{u}}=\mathrm{u} / \mathrm{c}, \tilde{\mathrm{w}}=\mathrm{w} / \mathrm{c}, \tilde{p}=p / \rho_{o} \mathrm{c}^{2}, \\
& \tilde{p}_{c}=p_{c} / \rho_{o} \mathrm{c}^{2}, \tilde{r}=r / a_{2}, \tilde{\mathrm{Q}}=\mathrm{Q} / \mathrm{c} a_{2}^{2}, \tilde{\lambda}_{1}=\lambda_{1} \mathrm{c} / a_{2}, \tilde{\lambda}_{2}=\lambda_{2} \mathrm{c} / a_{2}, \tilde{\mathrm{K}}=\mathrm{K} / \Phi a_{2}^{2} .
\end{aligned}
$$

and the dimensionless parameters

$$
\begin{aligned}
& \varepsilon=b / a_{2}, \alpha=2 \pi a_{2} / \lambda, \mathrm{R}=\rho_{o} \mathrm{c} a_{2} / \mu, \mathrm{k}_{\mathrm{n}}=\mathrm{A} / a_{2}, \mathrm{M}=\sqrt{a_{2} \sigma / \rho_{o} c} \mathrm{~B}_{o}^{2}, \\
& \delta=a_{1} / a_{2}, \text { and } \chi=k_{c} \rho_{o} \mathrm{c}^{2}=\mathrm{c}^{2} / \mathrm{c}_{o}^{2} ; \mathrm{c}_{o}=1 / \sqrt{k_{c} \rho_{o}} .
\end{aligned}
$$

which represent the amplitude ratio, wave number, Reynolds number, slip parameter, magnetic parameter, radius ratio, and the compressibility parameter, respectively. We disclose that although the compressibility $k_{c}$ is small, the dimensionless parameter $\chi$ has a magnitude of order 1 for the flows considered. In terms of these variables, equations (9) and (12) [if dropping the tilde signs] reduce to

$$
\begin{gathered}
\rho=\exp \left[\chi\left(p-p_{c}\right)\right], \\
\frac{\partial \rho}{\partial t}+\mathrm{u} \frac{\partial \rho}{\partial r}+\mathrm{w} \frac{\partial \rho}{\partial z}+\rho\left(\frac{\partial \mathrm{u}}{\partial r}+\frac{\mathrm{u}}{r}+\frac{\partial \mathrm{w}}{\partial z}\right)=0 \\
\left(1+\lambda_{1} \frac{\partial}{\partial t}\right)\left[\rho \frac{\partial u}{\partial t}+\rho\left(\mathrm{u} \frac{\partial \mathrm{u}}{\partial r}+\mathrm{w} \frac{\partial \mathrm{u}}{\partial z}\right)\right]=-\left(1+\lambda_{1} \frac{\partial}{\partial t}\right) \frac{\partial p}{\partial r}
\end{gathered}
$$




$$
\begin{aligned}
& +\frac{1}{\mathrm{R}}\left(1+\lambda_{2} \frac{\partial}{\partial t}\right)\left[\frac{\partial^{2} \mathrm{u}}{\partial r^{2}}+\frac{1}{r} \frac{\partial \mathrm{u}}{\partial r}-\frac{\mathrm{u}}{r^{2}}+\frac{\partial^{2} \mathrm{u}}{\partial z^{2}}+\frac{1}{3} \frac{\partial}{\partial r}\left(\frac{\partial \mathrm{u}}{\partial r}+\frac{\mathrm{u}}{r}+\frac{\partial \mathrm{w}}{\partial z}\right)\right] \\
& -\frac{1}{\mathrm{R} \mathrm{K}}\left(1+\lambda_{2} \frac{\partial}{\partial t}\right) \mathrm{u}-\mathrm{M}^{2}\left(1+\lambda_{1} \frac{\partial}{\partial t}\right) \mathrm{u}, \\
& \left(1+\lambda_{1} \frac{\partial}{\partial t}\right)\left[\rho \frac{\partial \mathrm{w}}{\partial t}+\rho\left(\mathrm{u} \frac{\partial \mathrm{w}}{\partial r}+\mathrm{w} \frac{\partial \mathrm{w}}{\partial z}\right)\right]=-\left(1+\lambda_{1} \frac{\partial}{\partial t}\right) \frac{\partial p}{\partial z} \\
& +\frac{1}{\mathrm{R}}\left(1+\lambda_{2} \frac{\partial}{\partial t}\right)\left[\frac{1}{r} \frac{\partial}{\partial r}\left(r \frac{\partial \mathrm{w}}{\partial r}\right)+\frac{\partial^{2} \mathrm{w}}{\partial z^{2}}+\frac{1}{3} \frac{\partial}{\partial z}\left(\frac{\partial \mathrm{u}}{\partial r}+\frac{\mathrm{u}}{r}+\frac{\partial \mathrm{w}}{\partial z}\right)\right] \\
& -\frac{1}{\mathrm{R} \mathrm{K}}\left(1+\lambda_{2} \frac{\partial}{\partial t}\right) \mathrm{w}-\mathrm{M}^{2}\left(1+\lambda_{1} \frac{\partial}{\partial t}\right) \mathrm{w} .
\end{aligned}
$$

while the boundary conditions (13) for $u$ at $r=R_{1}$ remain the same after scaling, where $\mathrm{R}_{1}$ and $\mathrm{R}_{2}$ in their dimensionless form read

$$
\mathrm{R}_{1}=\delta, \quad \mathrm{R}_{2}=1+\eta(z, t) .
$$

with $\eta(z, t)=\varepsilon \cos \alpha(z-t)$. Thus, the boundary conditions in their dimensionless form read

$$
\begin{gathered}
\mathrm{u}(\delta, z, t)=0, \quad \mathrm{w}(\delta, z, t)=0 . \\
\mathrm{u}(1+\eta(z, t), z, t)=\frac{\partial \eta(z, t)}{\partial t}, \quad \mathrm{w}(1+\eta(z, t), z, t)=\mathrm{k}_{\mathrm{n}} \frac{\partial \mathrm{w}(r, z, t)}{\partial \mathrm{r}} .
\end{gathered}
$$

From Eq. (15), the dimensionless flow rate will then be

$$
<\mathrm{Q}>=2 \pi \int_{\delta}^{1} \mathrm{w}(r, z, t) r d r .
$$

We note with interest that if $\mathrm{M}=0, \mathrm{k}_{\mathrm{n}}=0, \mathrm{~K} \rightarrow \infty$, and $\lambda_{2}=0$, Eqs. (19) to (22) reduce to that of non-slip flow [24], and if $\mathrm{M}=0, \mathrm{k}_{\mathrm{n}}=0, \mathrm{~K} \rightarrow \infty$, and $\delta=0$, we recover the governing equations of [15].

\section{Method of solution}

To illustrate the nature of solution we shall consider the important case of no flow in the absence of the peristaltic wave. As a consequence, the pressure $p$ is equal to the reference $p_{o}$ and we assume the solutions for the pressure, velocity and density in the form

$$
\begin{aligned}
& p=p_{o}+\varepsilon p_{1}(r, z, t)+\varepsilon^{2} p_{2}(r, z, t)+\ldots, \\
& \mathrm{u}=\varepsilon \mathrm{u}_{1}(r, z, t)+\varepsilon^{2} \mathrm{u}_{2}(r, z, t)+\ldots, \\
& \mathrm{w}=\varepsilon \mathrm{w}_{1}(r, z, t)+\varepsilon^{2} \mathrm{w}_{2}(r, z, t)+\ldots, \\
& \rho=1+\varepsilon \rho_{1}(r, z, t)+\varepsilon^{2} \rho_{2}(r, z, t)+\ldots,
\end{aligned}
$$

Substituting the expansions $(24)$ in $(18-20)$ and equating the coefficients of equal powers of $\varepsilon$ on both sides of the equations, we obtain for $\varepsilon$ :

$$
\begin{gathered}
\rho_{1}=\chi p_{1}, \\
\frac{\partial \rho_{1}}{\partial t}+\frac{\partial \mathrm{u}_{1}}{\partial r}+\frac{\mathrm{u}_{1}}{r}+\frac{\partial \mathrm{w}_{1}}{\partial z}=0
\end{gathered}
$$




$$
\begin{aligned}
& \left(1+\lambda_{1} \frac{\partial}{\partial t}\right) \frac{\partial \mathrm{u}_{1}}{\partial t}=-\left(1+\lambda_{1} \frac{\partial}{\partial t}\right) \frac{\partial p_{1}}{\partial r}+\frac{1}{\mathrm{R}}\left(1+\lambda_{2} \frac{\partial}{\partial t}\right)\left[\frac{\partial^{2} \mathrm{u}_{1}}{\partial r^{2}}+\frac{1}{r} \frac{\partial \mathrm{u}_{1}}{\partial r}-\frac{\mathrm{u}_{1}}{r^{2}}+\frac{\partial^{2} \mathrm{u}_{1}}{\partial z^{2}}\right] \\
& \left.+\frac{1}{3 \mathrm{R}}\left(1+\lambda_{2} \frac{\partial}{\partial t}\right) \frac{\partial}{\partial r}\left(\frac{\partial \mathrm{u}_{1}}{\partial r}+\frac{\mathrm{u}_{1}}{r}+\frac{\partial \mathrm{w}_{1}}{\partial z}\right)-\frac{1}{\mathrm{R} \mathrm{K}}\left(1+\lambda_{2} \frac{\partial}{\partial t}\right) \mathrm{u}_{1}-\mathrm{M}^{2}(1+\lambda)_{1} \frac{\partial}{\partial t}\right) \mathrm{u}_{1} \\
& \left(1+\lambda_{1} \frac{\partial}{\partial t}\right) \frac{\partial \mathrm{w}_{1}}{\partial t}=-\left(1+\lambda_{1} \frac{\partial}{\partial t}\right) \frac{\partial p_{1}}{\partial z}+\frac{1}{\mathrm{R}}\left(1+\lambda_{2} \frac{\partial}{\partial t}\right)\left[\frac{\partial^{2} \mathrm{w}_{1}}{\partial r^{2}}+\frac{1}{r} \frac{\partial \mathrm{w}_{1}}{\partial r}+\frac{\partial^{2} \mathrm{w}_{1}}{\partial z^{2}}\right] \\
& +\frac{1}{3 \mathrm{R}}\left(1+\lambda_{2} \frac{\partial}{\partial t}\right) \frac{\partial}{\partial z}\left(\frac{\partial \mathrm{u}_{1}}{\partial r}+\frac{\mathrm{u}_{1}}{r}+\frac{\partial \mathrm{w}_{1}}{\partial z}\right)-\frac{1}{\mathrm{R} \mathrm{K}}\left(1+\lambda_{2} \frac{\partial}{\partial t}\right) \mathrm{w}_{1}-\mathrm{M}^{2}\left(1+\lambda_{1} \frac{\partial}{\partial t}\right) \mathrm{w}_{1}
\end{aligned}
$$

And for $\varepsilon^{2}$ :

$$
\begin{gathered}
\rho_{2}=\chi p_{2}+\frac{1}{2} \chi^{2} p_{1}^{2}, \\
\frac{\partial \rho_{2}}{\partial t}+\frac{\partial \mathrm{u}_{2}}{\partial r}+\frac{\mathrm{u}_{2}}{r}+\frac{\partial \mathrm{w}_{2}}{\partial z}+\rho_{1}\left(\frac{\partial \mathrm{u}_{1}}{\partial r}+\frac{\mathrm{u}_{1}}{r}+\frac{\partial \mathrm{w}_{1}}{\partial z}\right)+\mathrm{u}_{1} \frac{\partial \rho_{1}}{\partial r}+\mathrm{w}_{1} \frac{\partial \rho_{1}}{\partial z}=0 \\
\left(1+\lambda_{1} \frac{\partial}{\partial t}\right)\left[\frac{\partial \mathrm{u}_{2}}{\partial t}+\rho_{1} \frac{\partial \mathrm{u}_{1}}{\partial t}+\mathrm{u}_{1} \frac{\partial \mathrm{u}_{1}}{\partial r}+\mathrm{w}_{1} \frac{\partial \mathrm{u}_{1}}{\partial z}\right]=-\left(1+\lambda_{1} \frac{\partial}{\partial t}\right) \frac{\partial p_{2}}{\partial r} \\
+\frac{1}{3 \mathrm{R}}\left(1+\lambda_{2} \frac{\partial}{\partial t}\right)\left[\frac{\partial^{2} \mathrm{u}_{2}}{\partial r^{2}}+\frac{1}{r} \frac{\partial \mathrm{u}_{2}}{\partial r}-\frac{\mathrm{u}_{2}}{r^{2}}+\frac{\partial^{2} \mathrm{u}_{2}}{\partial z^{2}}\right] \\
\left(1+\lambda_{1} \frac{\partial}{\partial t}\right)\left[\frac{\partial \mathrm{w}_{2}}{\partial t}+\rho_{1} \frac{\partial \mathrm{w}_{1}}{\partial t}+\mathrm{u}_{1} \frac{\partial \mathrm{w}_{1}}{\partial r}+\mathrm{w}_{1} \frac{\partial \mathrm{w}_{1}}{\partial z}\right]=-\left(1+\lambda_{1} \frac{\partial}{\partial t}\right) \frac{\partial p_{2}}{\partial z}\left(\frac{\partial \mathrm{u}_{2}}{\partial r}+\frac{\mathrm{u}_{2}}{r}+\frac{\partial \mathrm{w}_{2}}{\partial z}\right)-\frac{1}{\mathrm{R} \mathrm{K}}\left(1+\lambda_{2} \frac{\partial}{\partial t}\right) \mathrm{u}_{2}-\mathrm{M}^{2}\left(1+\lambda_{1} \frac{\partial}{\partial t}\right) \mathrm{u}_{2} \\
+\frac{1}{\mathrm{R}}\left(1+\lambda_{2} \frac{\partial}{\partial t}\right)\left[\frac{\partial^{2} \mathrm{w}_{2}}{\partial r^{2}}+\frac{1}{r} \frac{\partial \mathrm{w}_{2}}{\partial r}+\frac{\partial^{2} \mathrm{w}_{2}}{\partial z^{2}}\right]+\frac{1}{3 \mathrm{R}}\left(1+\lambda_{2} \frac{\partial}{\partial t}\right) \frac{\partial}{\partial z}\left(\frac{\partial \mathrm{u}_{2}}{\partial r}+\frac{\mathrm{u}_{2}}{r}+\frac{\partial \mathrm{w}_{2}}{\partial z}\right) \\
-\frac{1}{\mathrm{R} \mathrm{K}}\left(1+\lambda_{2} \frac{\partial}{\partial t}\right) \mathrm{w}_{2}-\mathrm{M}^{2}\left(1+\lambda_{1} \frac{\partial}{\partial t}\right) \mathrm{w}_{2},(26)^{2}
\end{gathered}
$$

etc. We represent the boundary conditions (21) and (22) by a Taylor expansion around $r=1$, then by the aid of the expansions (24) we express the sines and cosines in exponential powers and equate equal powers of $\varepsilon$ in both sides of the equations. We obtain for $\varepsilon$ :

$$
\begin{array}{cc}
\mathrm{u}_{1}(\delta, z, t)=0, & \mathrm{w}_{1}(\delta, z, t)=0 . \\
\mathrm{u}_{1}(1, z, t)=-\frac{i \alpha}{2}\left(e^{i \alpha(z-t)}-e^{-i \alpha(z-t)}\right), & \mathrm{w}_{1}(1, z, t)=\mathrm{k}_{\mathrm{n}} \mathrm{w}_{1 r}(1, z, t) .
\end{array}
$$

And for $\varepsilon^{2}$ :

$$
\mathrm{u}_{2}(\delta, z, t)=0, \quad \mathrm{w}_{2}(\delta, z, t)=\mathrm{k}_{\mathrm{n}} \mathrm{w}_{2 r}(\delta, z, t) .
$$




$$
\begin{aligned}
& \mathrm{u}_{2}(1, z, t)+\frac{1}{2}\left(e^{i \alpha(z-t)}+e^{-i \alpha(z-t)}\right) \mathrm{u}_{1 r}(1, z, t)=0, \\
& \mathrm{w}_{2}(1, z, t)+\frac{1}{2}\left(e^{i \alpha(z-t)}+e^{-i \alpha(z-t)}\right) \mathrm{w}_{1 r}(1, z, t)=0 .
\end{aligned}
$$

Finally, using the expansions (24) we represent the flow rate Q in Eq. (23) as

$$
<\mathrm{Q}>=2 \pi\left[\varepsilon \int_{\delta}^{1} \mathrm{w}_{1}(r, z, t) r d r+\varepsilon^{2} \int_{\delta}^{1} \mathrm{w}_{2}(r, z, t) r d r+O\left(\varepsilon^{3}\right)\right] .
$$

Examination of Eqs. $(25)$ with $(27,28)$ shows that a solution can be chosen in the form

$$
\begin{gathered}
\mathrm{u}_{1}(r, z, t)=U_{1}(r) e^{i \alpha(z-t)}+\bar{U}_{1}(r) e^{-i \alpha(z-t)}, \\
\mathrm{w}_{1}(x, y, t)=W_{1}(r) e^{i \alpha(z-t)}+\bar{W}_{1}(r) e^{-i \alpha(z-t)}, \\
p_{1}(r, z, t)=P_{1}(r) e^{i \alpha(z-t)}+\bar{P}_{1}(r) e^{-i \alpha(z-t)}, \\
\rho_{1}(r, z, t)=\chi P_{1}(r) e^{i \alpha(z-t)}+\chi \bar{P}_{1}(r) e^{-i \alpha(z-t)},
\end{gathered}
$$

And for equations $(26)$ with $(29,30)$ shows that a solution can be chosen in the form

$$
\begin{gathered}
\mathrm{u}_{2}(r, z, t)=U_{20}(r)+U_{2}(r) e^{2 i \alpha(z-t)}+\bar{U}_{2}(r) e^{-2 i \alpha(z-t)}, \\
\mathrm{w}_{2}(r, z, t)=W_{20}(r)+W_{2}(r) e^{2 i \alpha(z-t)}+\bar{W}_{2}(r) e^{-2 i \alpha(z-t)}, \\
p_{2}(r, z, t)=P_{20}(r)+P_{2}(r) e^{2 i \alpha(z-t)}+\bar{P}_{2}(r) e^{-2 i \alpha(z-t)}, \\
\rho_{2}(r, z, t)=D_{20}(r)+D_{2}(r) e^{2 i \alpha(z-t)}+\bar{D}_{2}(r) e^{-2 i \alpha(z-t)} .
\end{gathered}
$$

Here, the overbar denotes the complex conjugate.

Substituting (32) in $(25)$ and $(27,28)$, we obtain the following set of equations:

$$
U_{1}^{\prime}+\frac{U_{1}}{r}+i \alpha W_{1}=i \alpha \chi P_{1},
$$

$\left(-i \alpha+\mathrm{M}^{2}\right)\left(1-i \alpha \lambda_{1}\right) U_{1}=-\left(1-i \alpha \lambda_{1}\right) P_{1}^{\prime}$

$$
+\frac{\left(1-i \alpha \lambda_{2}\right)}{\mathrm{R}}\left[U_{1}^{\prime \prime}+\frac{U_{1}^{\prime}}{r}-\frac{U_{1}}{r^{2}}-\left(\alpha^{2}+\frac{1}{\mathrm{~K}}\right) U_{1}\right]+\frac{\left(1-i \alpha \lambda_{2}\right)}{3 \mathrm{R}} \frac{d}{d r}\left[U_{1}^{\prime}+\frac{U_{1}}{r}+i \alpha W_{1}\right] \text {, }
$$

$$
\begin{aligned}
& \left(-i \alpha+\mathrm{M}^{2}\right)\left(1-i \alpha \lambda_{1}\right) W_{1}=-i \alpha\left(1-i \alpha \lambda_{1}\right) P_{1} \\
& +\frac{\left(1-i \alpha \lambda_{2}\right)}{\mathrm{R}}\left[W_{1}^{\prime \prime}+\frac{W_{1}^{\prime}}{r}-\left(\alpha^{2}+\frac{1}{\mathrm{~K}}\right) W_{1}\right]+\frac{i \alpha\left(1-i \alpha \lambda_{2}\right)}{3 \mathrm{R}}\left[U_{1}^{\prime}+\frac{U_{1}}{r}+i \alpha W_{1}\right],
\end{aligned}
$$

$$
\begin{array}{ll}
U_{1}(\delta)=0, & W_{1}(\delta)=0, \\
U_{1}(1)=\frac{-i \alpha}{2}, & W_{1}(1)=\mathrm{k}_{\mathrm{n}} W_{1}^{\prime}(1),
\end{array}
$$

The set of equations for $\overline{U_{1}}, \overline{W_{1}}$, and $\overline{P_{1}}$ is conjugate to (34) so that it need not be written out explicitly. First, the system of equation (34) is solved. Substitution of (34) ${ }^{1}$ in $(34)^{2,3}$ yields

$$
\begin{aligned}
& -\gamma P_{1}^{\prime}+\left(U_{1}^{\prime \prime}+\frac{U_{1}^{\prime}}{r}-\frac{U_{1}}{r^{2}}-\beta^{2} U_{1}\right)=0, \\
& -\gamma P_{1}-\frac{i}{\alpha}\left(W_{1}^{\prime \prime}+\frac{W_{1}^{\prime}}{r}-\beta^{2} W_{1}\right)=0 .
\end{aligned}
$$

where the complex parameters $\gamma$ and $\beta$ are given by 


$$
\begin{gathered}
\gamma=\left(\frac{1-i \alpha \lambda_{1}}{1-i \alpha \lambda_{2}}\right) \mathrm{R}-\frac{i \alpha \chi}{3} \\
\beta^{2}=\alpha^{2}+\frac{1}{\mathrm{~K}}-\left(i \alpha-\mathrm{M}^{2}\right)\left(\frac{1-i \alpha \lambda_{1}}{1-i \alpha \lambda_{2}}\right) \mathrm{R} .
\end{gathered}
$$

With $W_{1}$ eliminated by means of $(34)^{1}$, we rewrite $(37)^{2}$ as

$$
\begin{aligned}
& -\frac{i \chi}{\alpha}\left[P_{1}^{\prime \prime}+\frac{P_{1}^{\prime}}{r}-\left(\beta^{2}+\frac{i \alpha \gamma}{\chi}\right) P_{1}\right] \\
& +\frac{1}{\alpha^{2}}\left(\frac{d}{d r}+\frac{1}{r}\right)\left(U_{1}^{\prime \prime}+\frac{U_{1}^{\prime}}{r}-\frac{U_{1}}{r^{2}}-\beta^{2} U_{1}\right)=0 .
\end{aligned}
$$

Differentiating (39) with respect to $r$ and using (37) ${ }^{1}$ to express the derivatives of $P_{1}$ in terms of $U_{1}$ and its derivatives, we are led to the following equation

$$
\begin{aligned}
-\frac{i \chi}{\alpha \gamma}\left(\frac{d^{2}}{d r^{2}}+\frac{1}{r} \frac{d}{d r}\right. & \left.-\frac{1}{r^{2}}-\left(\beta^{2}+\frac{i \alpha \gamma}{\chi}\right)\right)\left(U_{1}^{\prime \prime}+\frac{U_{1}^{\prime}}{r}-\frac{U_{1}}{r^{2}}-\beta^{2} U_{1}\right) \\
& +\frac{1}{\alpha^{2}}\left(\frac{d^{2}}{d r^{2}}+\frac{1}{r} \frac{d}{d r}-\frac{1}{r^{2}}\right)\left(U_{1}^{\prime \prime}+\frac{U_{1}^{\prime}}{r}-\frac{U_{1}}{r^{2}}-\beta^{2} U_{1}\right)=0 .
\end{aligned}
$$

which can be expressed as

$$
\mathrm{B}\left(\frac{d^{2}}{d r^{2}}+\frac{1}{r} \frac{d}{d r}-\frac{1}{r^{2}}-v^{2}\right)\left(\frac{d^{2}}{d r^{2}}+\frac{1}{r} \frac{d}{d r}-\frac{1}{r^{2}}-\beta^{2}\right) U_{1}=0,
$$

where the complex parameters $\mathrm{B}$ and $v$ are given by

$$
\mathrm{B}=1-\frac{i \alpha \chi}{\gamma}, \quad v^{2}=\beta^{2}-\frac{\left(\beta^{2}-\alpha^{2}\right)}{\mathrm{B}} .
$$

By using (38) and $(42)^{1}$, the expression in $(42)^{2}$ for $v$ can be written in terms of $\alpha, \mathrm{R}$, and $\chi$ as

$$
v^{2}=\alpha^{2} \frac{\left(1-\left(\chi+\frac{i \chi \mathrm{M}^{2}}{\alpha^{2}}\right)\right)\left(\frac{1-i \alpha \lambda_{1}}{1-i \alpha \lambda_{2}}\right) \mathrm{R}-\frac{4 i \alpha \chi}{3}-\frac{i \chi}{\alpha \mathrm{K}}}{\left(\frac{1-i \alpha \lambda_{1}}{1-i \alpha \lambda_{2}}\right) \mathrm{R}-\frac{4 i \alpha \chi}{3}} .
$$

Notice that if $\mathrm{M}=0, \lambda_{2}=0$, and $\mathrm{K} \rightarrow \infty$, then $v^{2}$ of Mekheimer and Abdel-Wahab [24] is recovered. Also notice that $v=\alpha$ for $\chi=0$ and $\mathrm{M}=0$, i.e. for incompressible liquids. The solution for $U_{1}$ with its boundary conditions is given by

$$
U_{1}(r)=C_{1} I_{1}(v r)+C_{2} I_{1}(\beta r)+C_{3} K_{1}(v r)+C_{4} K_{1}(\beta r)
$$

where $I_{1}$ and $K_{1}$ are the first-order modified Bessel functions of the first and second kind, respectively, and $\mathrm{C}_{1}, \mathrm{C}_{2}, \mathrm{C}_{3}$, and $\mathrm{C}_{4}$ are complex constants. Substitution of (44) into (37) ${ }^{1}$ yields

$$
\gamma P_{1}^{\prime}=C_{1}\left(v^{2}-\beta^{2}\right) I_{1}(v r)+C_{3}\left(v^{2}-\beta^{2}\right) K_{1}(v r),
$$

By using the properties $I_{1}(z)=I_{0}^{\prime}(z)$ and $K_{1}(z)=-K_{0}^{\prime}(z)$, where $I_{0}$ and $K_{0}$ are the zero-order modified Bessel functions of the first and second kind, respectively, it is easily seen that 


$$
P_{1}(r)=C_{5}+C_{1} \frac{\left(v^{2}-\beta^{2}\right)}{v \gamma} I_{0}(v r)-C_{3} \frac{\left(v^{2}-\beta^{2}\right)}{v \gamma} K_{0}(v r) .
$$

where $C_{5}$ is a complex constant. Substitution of (44) and (46) in (34) ${ }^{1}$, and using the properties $I_{1}^{\prime}(z)+I_{1}(z) / z=I_{0}(z)$ and $K_{1}^{\prime}(z)+K_{1}(z) / z=-K_{0}(z)$ we obtain

$$
W_{1}(r)=\frac{\delta_{1} C_{1}}{v} I_{0}(v r)+\frac{i \beta C_{2}}{\alpha} I_{0}(\beta r)-\frac{\delta_{1} C_{3}}{v} K_{0}(v r)-\frac{i \beta C_{4}}{\alpha} K_{0}(\beta r)+C_{5} \chi .
$$

Finally, by putting (46) into $(37)^{2}$ we deduce that

$$
C_{5}=0 \text {. }
$$

By using the boundary conditions (35) and (36) we get the constants $\mathrm{C}_{1}, \mathrm{C}_{2}, \mathrm{C}_{3}$, and $\mathrm{C}_{4}$ with the help of Mathematica 5.0 program. Thus, the first-order approximation of the velocity and pressure is described by the set of equations (44), (46) and (47). It should be pointed out that the expressions for $P_{1}(\mathrm{r})$ and $V_{1}(\mathrm{r})$ in [24] are incorrect. For the correct expressions, the above properties of the second-kind modified Bessel should be used, and which results in a negative sign multiplied by each of $\mathrm{C}_{3}$ and $\mathrm{C}_{4}$ in Eqs. $(3.22,3.23)$ of [24].

To solve the system of the second order of $\varepsilon$, we use Eq. (33) into Eqs. (26) and (29, 30 ), we then obtain the following set of equations:

$$
\begin{gathered}
D_{20}=\chi P_{20}+\chi^{2} \overline{P_{1}} P_{1}, \\
U_{20}^{\prime}+\frac{U_{20}}{r}=-\chi\left[P_{1} \overline{U_{1}^{\prime}}+\overline{P_{1}} U_{1}^{\prime}+\frac{P_{1} \overline{U_{1}}}{r}+\frac{\bar{P}_{1}}{r}+\overline{P_{1}^{\prime}} U_{1}+P_{1}^{\prime} \overline{U_{1}}\right], \\
-P_{20}^{\prime}+\frac{1}{\mathrm{R}}\left[U_{20}^{\prime \prime}+\frac{U_{20}^{\prime}}{r}-\left(\frac{1}{r^{2}}+n^{2}\right) U_{20}\right]+\frac{1}{3 \mathrm{R}} \frac{d}{d r}\left(U_{20}^{\prime}+\frac{U_{20}}{r}\right) \\
=i \alpha \chi P_{1} \overline{U_{1}}-i \alpha \chi \overline{P_{1}} U_{1}+U_{1} \overline{U_{1}^{\prime}}+U_{1}^{\prime} \overline{U_{1}}-i \alpha W_{1} \overline{U_{1}}+i \alpha \overline{W_{1}} U_{1}, \\
\frac{1}{\mathrm{R}}\left[W_{20}^{\prime \prime}+\frac{W_{20}^{\prime}}{r}-n^{2} W_{20}\right]=i \alpha \chi P_{1} \overline{W_{1}}-i \alpha \chi \overline{P_{1}} W_{1}+U_{1} \overline{W_{1}^{\prime}}+W_{1}^{\prime} \overline{U_{1}} . \\
U_{20}(\delta)=0, \overline{W_{20}}(\delta)=\mathrm{k}_{\mathrm{n}} W_{20}^{\prime}(\delta) . \\
U_{20}(1)+\frac{1}{2} \overline{U_{1}^{\prime}}(1)+\frac{1}{2} U_{1}^{\prime}(1)=0, \\
W_{20}(1)+\frac{1}{2} \overline{W_{1}^{\prime}}(1)+\frac{1}{2} W_{1}^{\prime}(1)=0 .
\end{gathered}
$$

It will be seen that, as far as the net flow is considered, only the functions $U_{20}, W_{20}$, $P_{20}$, and $D_{20}$ participate in the solution, as long as terms up to $\mathrm{O}\left(\varepsilon^{2}\right)$ are retained. Thus, the functions $U_{2}, W_{2}, P_{2}$, and $D_{2}$ do not contribute to the net flow, and therefore, we shall not write down the equations that these functions satisfy, nor solve them. It should be noted that Eqs $(3.25,3.26)$ in [24] and Eqs. $(3.34,3.35)$ in [8] are incorrect. In fact, for the correct expressions the term $\left(1-i \alpha t_{m}\right)$ should be 1 . We continue with the solutions for $P_{20}, W_{20}$, and $U_{20}$.

We rewrite the Eqs. $(49)^{2,3,4}$ as

$$
\begin{aligned}
& U_{20}^{\prime}+\frac{U_{20}}{r}=-\chi H, \\
& -P_{20}^{\prime}+\frac{4}{3 \mathrm{R}}\left[U_{20}^{\prime \prime}+\frac{U_{20}^{\prime}}{r}-\left(\frac{1}{r^{2}}+\frac{3}{4} n^{2}\right) U_{20}\right]=F, \\
& W_{20}^{\prime \prime}+\frac{W_{20}^{\prime}}{r}-n^{2} W_{20}=\mathrm{R} G \text {. }
\end{aligned}
$$


where the functions $H, F$, and $G$, corresponding to the right-hand sides of $(6.49)^{2,3,4}$ are given by

$$
\begin{gathered}
H=\frac{1}{r} \frac{d}{d r}\left[r\left(P_{1} \overline{U_{1}}+\bar{P}_{1} U_{1}\right)\right], \\
F=i \alpha \chi P_{1} \overline{U_{1}}-i \alpha \chi \overline{P_{1}} U_{1}+U_{1} \overline{U_{1}^{\prime}}+U_{1}^{\prime} \overline{U_{1}}-i \alpha W_{1} \overline{U_{1}}+i \alpha \overline{W_{1}} U_{1}, \\
G=\frac{1}{r} \frac{d}{d r}\left[r\left(U_{1} \overline{W_{1}}+W_{1} \overline{U_{1}}\right)\right] .
\end{gathered}
$$

with $n^{2}=1 \backslash \mathrm{K}+\mathrm{M}^{2} \mathrm{R}$. It should be noted that the differential equation (3.35) of [8] resembles, after simplification, our eq. $(52)^{3}$, but with $\mathrm{M}=0$, which is a modified Bessel differential equation and therefore should have the same solution given here and not the ones in [1, 15, 24, 39]. The same goes for eq. (15) in [7].

Here, Eqs. (34) ${ }^{3}$ is used to eliminate $P_{1}$ in function $G$. With $H$ given by $(53)^{1}$, it is easily seen that the solution for $U_{20}$ is given by

$$
U_{20}(r)=\frac{D_{1}}{r}-\chi\left(P_{1}(r) \bar{U}_{1}(r)+\bar{P}_{1}(r) U_{1}(r)\right) .
$$

where the complex constant $D_{1}$ follows from the boundary condition (51). It should be noted that the factor $\left(1-i \alpha t_{m}\right)$ in Eq. (3.28) $)^{2,3}$ of [24] is incorrect and should be 1 .

Elimination of $U_{1}{ }^{\prime}$ by means of (34) ${ }^{1}$ and by using (35) and (36) leads to

$$
D_{1}=\frac{-i \alpha}{2} \mathrm{k}_{\mathrm{n}}\left(\overline{W_{1}^{\prime}}(1)-W_{1}^{\prime}(1)\right) \text {. }
$$

Notice that $D_{1}=0$ at $\mathrm{k}_{\mathrm{n}}=0$ as in $[1,8,15,24,39]$, in addition, $U_{20}(r)=0$ for $\chi=0$, i.e., for incompressible liquids. With $G$ given by $(53)^{3}$, it is easily seen that the solution for $W_{20}$ is given by

$W_{20}(r)=D_{2} I_{o}(n r)+D_{3} K_{o}(n r)+\mathrm{R} I_{o}(n r) \int_{\delta}^{r} S_{1}(y) d y-\mathrm{R} K_{o}(n r) \int_{\delta}^{r} S_{2}(y) d y$,

where

$$
S_{1}(y)=K_{o}(n y)\left(\zeta(y)+y \zeta^{\prime}(y)\right), S_{2}(y)=I_{o}(n y)\left(\zeta(y)+y \zeta^{\prime}(y)\right),
$$

with

$$
\zeta(r)=\left(U_{1}(r) \overline{W_{1}}(r)+W_{1}(r) \overline{U_{1}}(r)\right) .
$$

The constants of integration $D_{2}$ and $D_{3}$ are defined as

such that

$$
\begin{aligned}
& D_{2}=\frac{1}{L_{1}}\left\{\mathrm{R} \beta_{1} I_{o}(n)-\mathrm{R} \beta_{2} K_{o}(n)+W_{20}(1)\right\}, \\
& D_{3}=\frac{1}{L_{2}}\left\{\mathrm{R} \beta_{1} I_{o}(n)-\mathrm{R} \beta_{2} K_{o}(n)+W_{20}(1)\right\} .
\end{aligned}
$$

and

$$
\beta_{1}=\int_{1}^{\delta} S_{1}(y) d y, \beta_{2}=\int_{1}^{\delta} S_{2}(y) d y
$$

$$
\begin{aligned}
& L_{1}=\frac{I_{o}(n)\left(K_{o}(n \delta)+n \mathrm{k}_{\mathrm{n}} K_{1}(n \delta)\right)-K_{o}(n)\left(I_{o}(n \delta)-n \mathrm{k}_{\mathrm{n}} I_{1}(n \delta)\right)}{K_{o}(n \delta)+n \mathrm{k}_{\mathrm{n}} K_{1}(n \delta)}, \\
& L_{2}=-\frac{I_{o}(n)\left(K_{o}(n \delta)+n \mathrm{k}_{\mathrm{n}} K_{1}(n \delta)\right)-K_{o}(n)\left(I_{o}(n \delta)-n \mathrm{k}_{\mathrm{n}} I_{1}(n \delta)\right)}{I_{o}(n \delta)-n \mathrm{k}_{\mathrm{n}} I_{1}(n \delta)} .
\end{aligned}
$$

Then $W_{20}$ can be simplified as 


$$
\begin{aligned}
W_{20}(r)=\mathrm{R} & \beta_{1}\left[\left(\frac{I_{o}(n)}{L_{1}} I_{o}(n r)+\frac{I_{o}(n)}{L_{2}} K_{o}(n r)\right)+I_{o}(n r) \int_{\delta}^{r} S_{1}(y) d y\right] \\
& -\mathrm{R} \beta_{2}\left[\left(\frac{K_{o}(n)}{L_{1}} I_{o}(n r)+\frac{K_{o}(n)}{L_{2}} K_{o}(n r)\right)\right. \\
& \left.+K_{o}(n r) \int_{\delta}^{r} S_{2}(y) d y\right]+W_{20}(1)\left(\frac{I_{o}(n)}{L_{1}}+\frac{K_{o}(n)}{L_{2}}\right) .
\end{aligned}
$$

Finally, it is easily seen that the solution for $P_{20}$ is given by

$$
P_{20}(r)=D_{4}-\frac{4 \chi}{3 \mathrm{R}} H(r)-\int_{\delta}^{r} F_{1}(y) \mathrm{dy} .
$$

where

$$
\begin{aligned}
F_{1}= & \left(i \alpha-\frac{n^{2}}{r}\right) \chi P_{1} \overline{U_{1}}-\left(i \alpha+\frac{n^{2}}{r}\right) \chi \overline{P_{1}} U_{1}+U_{1} \overline{U_{1}^{\prime}} \\
& +U_{1}{ }^{\prime} \overline{U_{1}}-i \alpha W_{1} \overline{U_{1}}+i \alpha \overline{W_{1}} U_{1} .
\end{aligned}
$$

Here, the complex constant $D_{4}=P_{20}(\delta)+4 \chi H(\delta) / 3 \mathrm{R}$ follows from a given pressure $P_{20}(\delta)$ on the inner tube of the annulus. Note that if $n^{2}=0$ (that is; $\mathrm{M}=0$ and $\mathrm{K} \rightarrow \infty$ ), $P_{20}$ will be the same as [24]. It should be noted that the factor $\left(1-i \alpha t_{m}\right)$ in Eqs. (3.32, $3.33,3.34$ ), and in the constants $D_{2}, D_{3}$, and $D_{4}$ of [24] is incorrect and should be 1 . The same applies for Eq. (31), Eq. (3.40), and the solution of $V_{20}$ of [7], [8], and [39], respectively. Further, the integral in Eq. (3.34) of [24] should be for $F(y)$ rather than $\zeta(y)$.

Next, the net flow is considered which is the flow averaged over one period of time. We introduce the average of a variable $g$ over one period $T$ of time $t$ as

$$
<g>=\frac{1}{T} \int_{0}^{T} g(r, z, t) d t,
$$

where $T=2 \pi / \alpha$. Consequently, the net axial velocity $\langle w\rangle$ reads

$$
<w>=\varepsilon^{2} W_{20}(r),
$$

By neglecting the terms of $O\left(\varepsilon^{3}\right)$, the net flow rate $<Q>$ is given by

$$
<Q>=2 \pi \varepsilon^{2} \int_{\delta}^{1} W_{20}(r) r d r .
$$

Thus, the travelling wave induces a net flow of the liquid, of which the (dimensionless) rate is expressed by (6.61). Hence, the net flow is an effect of order $\varepsilon^{2}$. It should be noted that the factor $\left(1-i \alpha t_{m}\right)$ in Eq. (4.1), Eq. (36), Eq. (4.1), and Eq. (16) of [24], [7], [8], and [39], respectively, is incorrect and should be 1 .

\section{Numerical results and discussion}

Our primary interest in this model is to study the effects of the radius ratio $\delta$, nonNewtonian parameters $\lambda_{1}$ and $\lambda_{2}$, compressibility $\chi$, Reynolds number $\mathrm{R}$, magnetic parameter $\mathrm{M}$, permeability $\mathrm{K}$, and slip parameter $\mathrm{k}_{\mathrm{n}}$ on the net flow rate $\langle\mathrm{Q}>$ though an annulus with peristaltic mechanism on the outer wall.

It is obvious that we have to choose $(\varepsilon<1)$ because we have used the perturbation method with the amplitude ratio $(\varepsilon)$ as a parameter. We consider the net flow rate given by (61). After integrating by parts, $<\mathrm{Q}>$ can be expressed as 


$$
\begin{aligned}
<Q>= & 2 \pi \varepsilon^{2} \mathrm{R}\left[\left(\beta_{1} \frac{I_{o}(n)}{L_{1}}-\beta_{2} \frac{K_{o}(n)}{L_{1}}\right) \int_{\delta}^{1} r I_{o}(n r) d r\right. \\
& \left.+\left(\beta_{1} \frac{I_{o}(n)}{L_{2}}-\beta_{2} \frac{K_{o}(n)}{L_{2}}\right) \int_{\delta}^{1} r K_{o}(n r) d r+I_{3}-I_{4}\right] \\
& +2 \pi \varepsilon^{2} W_{20}(1)\left[\frac{1}{L_{1}} \int_{\delta}^{1} r I_{o}(n r) d r+\frac{1}{L_{2}} \int_{\delta}^{1} r K_{o}(n r) d r\right],
\end{aligned}
$$

where $I_{3}$ and $I_{4}$ are given by

$$
\begin{gathered}
I_{3}=\frac{\beta_{1}}{2} \frac{{ }_{0} F_{1}\left(2 ; \frac{n^{2}}{4}\right)}{\sqrt{2}}-\frac{1}{2} \int_{\delta}^{1} r^{2} \frac{{ }_{0} F_{1}\left(2 ; \frac{n^{2}}{4}\right)}{\sqrt{2}} S_{1}(r) d r \\
I_{4}=-\frac{\beta_{2}}{n} K_{1}(n)+\frac{1}{n} \int_{\delta}^{1} r K_{1}(n r) S_{2}(r) d r,
\end{gathered}
$$

with $_{0} F_{1}\left(2 ; \frac{n^{2}}{4}\right) \backslash \sqrt{2}$ defined as the regularized confluent hypergeometric function for fixed $n$.

Here, the solution (57) for $W_{20}(r)$ is used. For the flow in an annulus, we compare our results to that of Mekheimer and Abdel-Wahab [24] which present the flow rate of compressible Maxwell fluid in an annulus in the absence of $\mathrm{M}, \mathrm{k}_{\mathrm{n}}$, and $\mathrm{K}$. We also compare our results to that of the authors in references $[1,7,8,15]$, which present the flow rate of a compressible non-Newtonian fluid in a tube, and we discuss the results numerically and graphically. Following [1] and [39], the value of $\chi$ is chosen between 0 and 1 in all the graphs. It is observed that viscoelastic behavior is more pronounced at great values of $\delta$. It is also seen that for large values of $\lambda_{1}$ the system exhibits viscoelastic behavior, while for small values of $\lambda_{1}$ the conventional viscous effects dominate. The situation is reversed in case of $\lambda_{2}$ for a fixed value of $\lambda_{1}$.

Now, we start to explain the effect of radius ratio $\delta$ on the flow rate for the annulus and the tube. In Figs. $2 \mathrm{a}$ and $2 \mathrm{~b}$, we investigate the dependence of the dimensionless flow rate $\langle\mathrm{Q}>$ on the compressibility parameter $\chi$ to compare the behavior of Newtonian, Maxwell, and Jeffrey fluids for a fixed value of R. We compare the flow pattern for $\delta=$ 0.001 and $\delta=0.2$ at $\varepsilon=0.001, \mathrm{R}=5000, \alpha=0.001, \mathrm{M}=0.001, \mathrm{k}_{\mathrm{n}}=0.05$, and $\mathrm{K}=$ 0.1 . For the curve $\lambda_{1}=0$ with $\lambda_{2}=0$ (Newtonian limiting) at $\delta=0.001$, we notice a deviation in the flow rate going towards negative values, which means a backflow. The same curve attains positive values for $\delta=0.2$. For the two curves $\lambda_{1}=5000$ with $\lambda_{2}=$ 0 (non-Newtonian Maxwell fluid) and $\lambda_{1}=5000$ with $\lambda_{2}=1000$ (Jeffrey fluid), there is a noticeable change in the plot, where the flow rate values for the Maxwell fluid is smaller than that of Jeffrey's at $\delta=0.001$. And, although the flow rate values for both curves increase with an increase in $\delta$, they coincide at a certain value of $\chi$ at $\delta=0.2$. The dependence of the flow rate on $\delta$ indicates that $\langle\mathrm{Q}>$ increases with an increase in $\delta$, accordingly, the flow rate for an annulus is more than that of a tube as shown in Fig. 2b. It is interesting to note that for $\delta=0$, the results of the tube-like structure are recovered. Moreover, setting $\mathrm{M}=0, \mathrm{k}_{\mathrm{n}}=0, \mathrm{~K} \rightarrow \infty$, and $\lambda_{2}=0$, the results of the annulus in [24] are recovered.

For the flow in an annulus, we compare the behavior of the Newtonian, Maxwell, and Jeffrey fluids for different values of R. The values of R in Figs. 2a and 2c are 5000 and 2000 , respectively. Figure 2a shows that for small values of $\chi$, the net flow rate in case of Maxwell fluid is less than that of Jeffrey fluid. Moreover, the net flow rate attains a 
minimum value in case of Newtonian fluid when compared to Maxwell and Jeffrey fluids. The noticeable fact is that both Newtonian and Jeffrey fluids attain a minimum value and then increase as $\chi$ increases, but this feature in absent in the Maxwell fluid. Figure $2 \mathrm{c}$ shows that the flow rate for the Newtonian fluid decays rapidly as compared to Maxwell and Jeffrey fluids, however, they all remain constant over a certain range of $\chi$ $(\chi \geq 0.5)$. It is shown that the flow rate, for the three curves, has positive values over the whole range of $\chi$. It is interesting to note that for $\chi<0.15$, the values of $<\mathrm{Q}>$ for the Jeffrey fluid are larger than that of Maxwell's. It is clear from Figs. 2a and 2c that for fixed values of $\lambda_{1}$ and $\lambda_{2}$ if Reynolds number is small enough, the curves of Maxwell and Jeffrey fluids coincide within a plotting accuracy but as Reynolds number increases the difference between the curves become more prominent. In other words, for fixed value of $\mathrm{R}$, if $\lambda_{1}$ and $\lambda_{2}$ are small the difference between Maxwell and Jeffrey fluids is less prominent as shown in Fig. 2d. Note that, while much of hemodynamics in a healthy human body has low Reynolds number resulting in a laminar flow, relatively high Reynolds number flows are observed at some specific locations which can cause transition to turbulence, e.g. the Reynolds number in the human aorta $[16,19]$.

In Fig. 3a, the dependence of $\langle\mathrm{Q}\rangle$ on the compressibility parameter is displayed for various values of $\mathrm{R}$. It is observed that $\mathrm{R}$ has an increasing effect on $\langle\mathrm{Q}\rangle$. In particular, for $\chi=0,<\mathrm{Q}>$ is weakly affected by $\mathrm{R}$. For $\chi>0$, however, $\langle\mathrm{Q}>$ strongly depends on $\mathrm{R}$. We notice that at any value within $0<\chi \leq 0.15$ (approximately), the net flow rate decreases rapidly until a certain value of $\chi$ where the flow becomes steady. Thus, the compressibility parameter $\chi$ has a significant influence on the net flow rate, and the Reynolds number $\mathrm{R}$ plays a more significant role in the net flow of a compressible liquid than that of an incompressible one. It is interesting to note that in order to compare our results with the ones in [15] we set $\mathrm{M}=0, \mathrm{k}_{\mathrm{n}}=0, \mathrm{~K} \rightarrow \infty$, and $\delta=0$ which corroborates the validity of our numerical codes.

Figure $3 \mathrm{~b}$ represents the variation of $\langle\mathrm{Q}\rangle$ with wave number $\alpha$ for various values of $\mathrm{R}$. It is shown that $<\mathrm{Q}>$ decreases with an increase in $\mathrm{R}$. We notice that for $\mathrm{R}=1000$ and $\mathrm{R}=10000$, the flow rate changes in the sense that it attains lower values as $\alpha$ increases, and specifically for $\mathrm{R}=10000$, the values of $\langle\mathrm{Q}\rangle$ tend to slightly rise up again after reaching the minima of the curve. However, for $\mathrm{R}=100$, the curve tends to deviate slightly attaining also negative values and then no change is observed, i.e. it becomes constant.

In order to illustrate the dependence of $\langle\mathrm{Q}>$ on $\chi$ for various values of the magnetic parameter M, we prepared Fig. 4a. It is noticed that $\langle\mathrm{Q}\rangle$ increases with an increase in M. Furthermore, at $\chi=0,<\mathrm{Q}>$ is weakly affected by M. For $\chi>0,<\mathrm{Q}>$ attains a minimum for a certain value of $\chi$ and then no change is observed.

Figure $4 \mathrm{~b}$ elucidates the variations of $\langle\mathrm{Q}\rangle$ with $\alpha$ for different values of $\mathrm{M}$. It is noticed that $\langle\mathrm{Q}\rangle$ is weakly affected by $\mathrm{M}$ at low values of $\alpha \quad(0 \leq \alpha \leq 0.002$ approximately). It is observed that for all the values of $\alpha>0.002,<\mathrm{Q}>$ has negative values, i.e., we observe a backflow. We notice that for each curve $\langle\mathrm{Q}\rangle$ attains a minimum value for a certain value of $\alpha$, and this minimum decreases with an increase in M. In particular, for $\mathrm{M}=0.002,\langle\mathrm{Q}\rangle$ attains a minimum value of $-2.36686 \times 10^{-3}$. Furthermore, the minima of the curves for the flow rate are shifting towards larger $\alpha$ as the magnetic parameter increases. From the above discussion, we observe that $\langle\mathrm{Q}\rangle$ increases with an increase in $\mathrm{M}$.

Figure 5a depicts the variations of $\langle\mathrm{Q}\rangle$ with $\chi$ for various values of the permeability parameter $\mathrm{K}$. It is shown that the permeability $\mathrm{K}$ has an increasing effect on $\langle\mathrm{Q}\rangle$, and this happens because the permeability parameter allows more fluid to pass through the pores. On the other hand, the flow rate decreases monotonically as the compressibility parameter increases. Moreover, at very low compressibility $(\chi=0)$, the 
net flow rate is weakly affected by $\mathrm{K}$. It is also observed that for $\mathrm{K}=0.5$ and $\mathrm{K} \rightarrow \infty$, $<\mathrm{Q}>$ remains constant over a certain range of $\chi$ and this range increases by increasing $\chi$.

In Fig. $5 \mathrm{~b}$, the net flow rate is plotted versus $\alpha$ for various values of $\mathrm{K}$. It is noticed that $\langle\mathrm{Q}\rangle$ is weakly affected by $\mathrm{K}$ at low values of $\alpha(0 \leq \alpha \leq 0.0015$ approximately), while for all the values of $\alpha>0.0015,<\mathrm{Q}>$ possesses negative values, i.e., we observe a backflow. It is shown that $\langle\mathrm{Q}\rangle$ increases with an increase in $\mathrm{K}$ and this happens because the permeability parameter allows more fluid to pass through the pores. Furthermore, it is observed the flow rate decreases monotonically as the wave number increases. For the two curves corresponding to $\mathrm{K}=0.5$ and $\mathrm{K} \rightarrow \infty$, it is noticed that both of them coincide within a plotting accuracy. It is interesting to note that in order to check the consistency of our results with the ones in [8], we set $\mathrm{M}=0, \mathrm{k}_{\mathrm{n}}=0, \lambda_{2}=0$, and $\delta=0$ which corroborates the validity of our numerical codes.

Figure 6 a shows the effects of Knudsen number $\mathrm{k}_{\mathrm{n}}$ on the net flow rate $\langle\mathrm{Q}>$ when plotted against $\chi$. It is clear that the flow rate changes in the sense that it decays rapidly as $\chi$ increases until reaching the minima of each curve at a specific value of $\chi$ (at $\chi=0.25$ for $\mathrm{k}_{\mathrm{n}}=0$, at $\chi=0.3$ for $\mathrm{k}_{\mathrm{n}}=0.05$ and $\mathrm{k}_{\mathrm{n}}=0.1$ ), where the values of $\langle\mathrm{Q}\rangle$ starts to increase. It is observed that at $\chi=0.07$, the net flow rate is weakly affected by $\mathrm{k}_{\mathrm{n}}$ since the values of $\langle\mathrm{Q}\rangle$ lie within the range $\left(1.13571 \times 10^{-6}-2.27986 \times 10^{-6}\right)$. It is shown that $<\mathrm{Q}>$ decreases with an increase in $\mathrm{k}_{\mathrm{n}}$ for $0<\chi \leq 0.625$, and that each curve reaches a minimum value before it starts to increase towards the largest $\chi$ within the indicated range. Moreover, we notice that $\langle\mathrm{Q}>$ has more magnitude for the slip fluid when compared with the nonslip fluid within the same range, and that for $\mathrm{k}_{\mathrm{n}}=0.05$ and $\mathrm{k}_{\mathrm{n}}=0.1$, the curves coincide within a plotting accuracy. For $0.625<\chi \leq 1$, it is observed that the net flow rate increases with an increase in $\mathrm{k}_{\mathrm{n}}$, and that $\langle\mathrm{Q}>$ has less magnitude for the slip fluid when compared with the nonslip fluid. We notice a deviation in the flow rate going towards negative values, which means a backflow.

In order to illustrate the dependence of the flow rate $<\mathrm{Q}>$ on the parameter $\alpha$ for different values of $k_{n}$, we prepared Fig. $6 b$. It is shown that $k_{n}$ has decreasing effect on $<\mathrm{Q}>$ within the indicated range of $\alpha$. It is also shown that $\langle\mathrm{Q}>$ is weakly affected by $\mathrm{k}_{\mathrm{n}}$ within $0<\alpha \leq 0.002$. It is noticed that $\langle\mathrm{Q}>$ goes to negative values, which indicates to a backflow. For $\mathrm{k}_{\mathrm{n}}=0$ and $\mathrm{k}_{\mathrm{n}}=0.05$, the flow rate decreases monotonically as $\alpha$ increases unlike the curve of $\mathrm{k}_{\mathrm{n}}=0.01$; here the flow rate values tend to reach its minimum at $\alpha=0.008$ where its value starts to increase again with an increase in $\alpha$. It is noticed that the magnitude of $\langle\mathrm{Q}\rangle$ for the nonslip flows is less than that of slip flows for the range of $\alpha$ considered. Note that if we set $\mathrm{M}=0, \lambda_{2}=0$, and $\delta=0$ in our numerical codes, the results of [7] are recovered.

In Figs. $7 \mathrm{a}$ and $7 \mathrm{~b}$, the dependence of $\langle\mathrm{Q}\rangle$ on the compressibility parameter is displayed for various values of the retardation time $\lambda_{2}$ for both the annulus and the tube. It is noticed that $\left\langle\mathrm{Q}>\right.$ decreases with an increase in $\lambda_{2}$ and that it is weakly affected by $\lambda_{2}$ for $\chi=0$. For $\lambda_{2}=0$ (Maxwell fluid) and $\lambda_{2}=100$ (Jeffrey fluid), it is shown that the flow rate coincides within a plotting accuracy and that it attains positive values. However, for $\lambda_{2}=1000$, the flow rate decays rapidly possessing negative values over a certain range of $\chi(0.15<\chi \leq 0.35)$ but increases again as $\chi$ increases $(0.35<\chi \leq 1)$. It is interesting to note that for large values of $\lambda_{2}$, Jeffrey fluid possesses the characteristics of Newtonian fluid [15], i.e., it decays rapidly as the compressibility parameter increases. The phenomenon brought by introducing the retardation time 
investigated. Further, the retardation time has an increasing effect on the flow rate in a tube as shown in Fig. 7b.

Figure 7c represents the net flow rate $\langle\mathrm{Q}>$ versus the wave number $\alpha$ for different values of $\lambda_{2}$ and two values of the radius ratio $\delta$, the curves are plotted for the interval of variation of $\alpha$ up to 0.01 . It is clear that for $\alpha=0$ that the flow rate is weakly affected by $\alpha$. Furthermore, for the deeply non-Newtonian regime (the solid curve), the flow exhibit viscoelastic behavior at $\delta=0.2$ and a highly oscillatory pattern (appearance of numerous maxima in the behavior of the physical variable) appears for the values of $\alpha$ larger than 0.002. It is quite obvious that $\langle\mathrm{Q}\rangle$ attains negative values over the indicated range of $\alpha$ (backflow) and increases with an increase in $\alpha$. The negative flow rate is interpreted as follows: the flow occurs in the direction opposite to the direction of propagation of traveling wave on the annulus wall, and as the annulus radius ratio increases, less backflow occurs at certain values of $\alpha$. However, the fluid flow by a peristaltic mechanism in the opposite direction should be attributed to a complicated, nonlinear form of the response of a Jeffrey fluid to the stress exerted by the wave. We confirm our results by studying the effect of $\delta$ on the flow rate $<\mathrm{Q}>$ for $\lambda_{2}=1000$. At $\delta=0.2$ (the dashed curve), the flow rate values are larger than that of $\delta=0.001$ (the dot-dashed curve). From the above discussion, we observe that there is a rapid decrease in the flow rate, and that it increases by increasing $\delta$ (backflow decreases). It is interesting to note that $\langle\mathrm{Q}>$ is negative and has highly oscillatory behavior for Maxwell fluid, which is in agreement with the results of [39]. This proves that oscillation decay depends upon the retardation time occurring in the constitutive equation of the Jeffrey fluid unlike that of the Maxwell fluid model [15].

In order to investigate novelties brought about by the introduction of radius ratio into the model, we plot Fig. 7d for a larger value of $\delta$. It is observed that as $\delta$ increases, the viscoelastic behavior becomes more pronounced at small values of the wave number $\alpha$. On the other hand, the behavior of Jeffrey fluid is less oscillatory when compared with the Maxwell fluid and vanishes for larger values of $\delta$. It is further observed that the net flow rate increases by increasing $\delta$. This provides a clear-cut indication to the fact that oscillation decay is highly dependent upon the radius ratio occurring in our Jeffrey model. Such kind of decay is not possible in the tube-like structure of [15], because we have included the non-Newtonian effects into the flow through an annulus with peristaltic mechanism on the outer wall by using a Jeffrey fluid model. Finally, we note that if $\mathrm{M}=$ $0, \delta=0, \mathrm{k}_{\mathrm{n}}=0$, and $\mathrm{K} \rightarrow \infty$ the results of [15] are recovered.
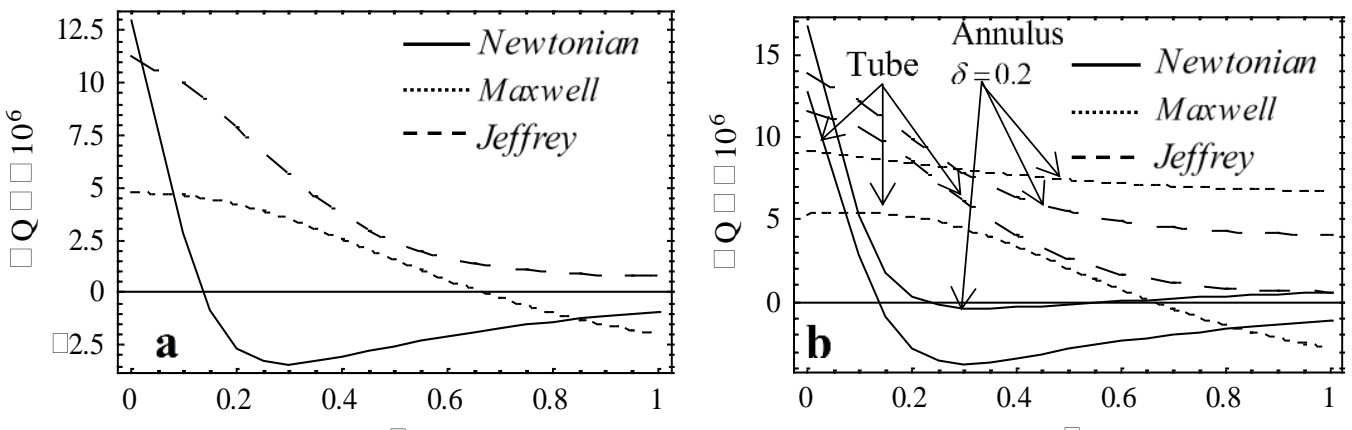

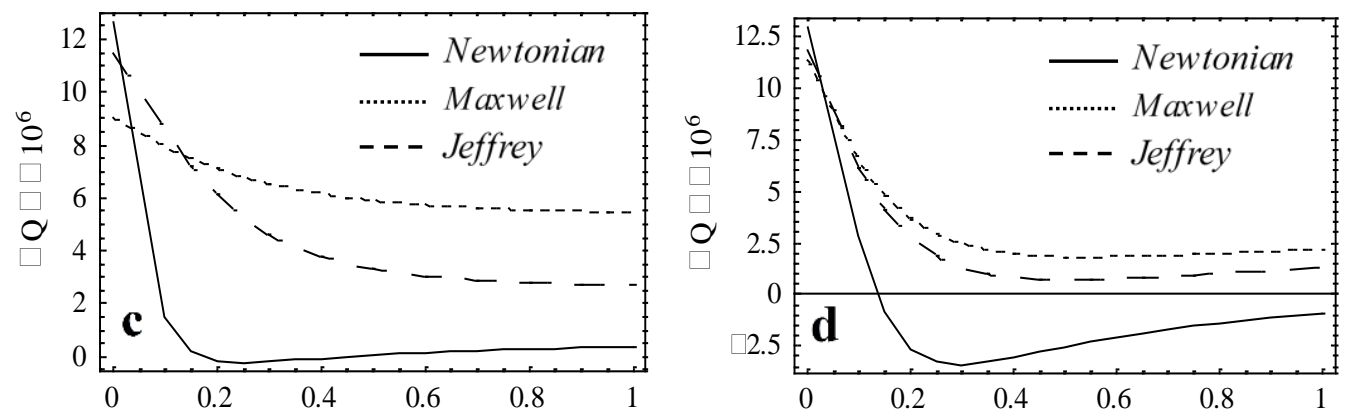

Fig. 2 Plot showing the dimensionless flow rate $<\mathrm{Q}>$ versus $\chi$ for: $\mathrm{R}=5000, \mathrm{M}=0.001, \mathrm{k}_{\mathrm{n}}=0.05$, $\mathrm{K}=0.1, \delta=0.001, \alpha=0.001$, and $\varepsilon=0.001$. For Maxwell fluid $\lambda_{1}=5000$ and for Jefrrey fluid $\lambda_{1}=5000, \lambda_{2}=1000$ (panel a); $\mathrm{R}=5000, \mathrm{M}=0.001, \mathrm{k}_{\mathrm{n}}=0.05, \mathrm{~K}=0.1, \alpha=0.001$, and $\varepsilon=0.001$. For Maxwell fluid $\lambda_{1}=5000$ and for Jefrrey fluid $\lambda_{1}=5000, \lambda_{2}=1000$ (panel b); $\mathrm{R}=2000, \mathrm{M}=0.001, \mathrm{k}_{\mathrm{n}}=0.05, \mathrm{~K}=0.1, \delta=0.001, \alpha=0.001$, and $\varepsilon=0.001$. For Maxwell fluid $\lambda_{1}=5000$ and for Jefrrey fluid $\lambda_{1}=5000, \lambda_{2}=1000$ (panel c); $\mathrm{R}=5000, \mathrm{M}=0.001, \mathrm{k}_{\mathrm{n}}=0.05$, $\mathrm{K}=0.1, \delta=0.001, \alpha=0.001$, and $\varepsilon=0.001$. For Maxwell fluid $\lambda_{1}=1000$ and for Jefrrey fluid $\lambda_{1}=1000, \lambda_{2}=200$ (panel d).
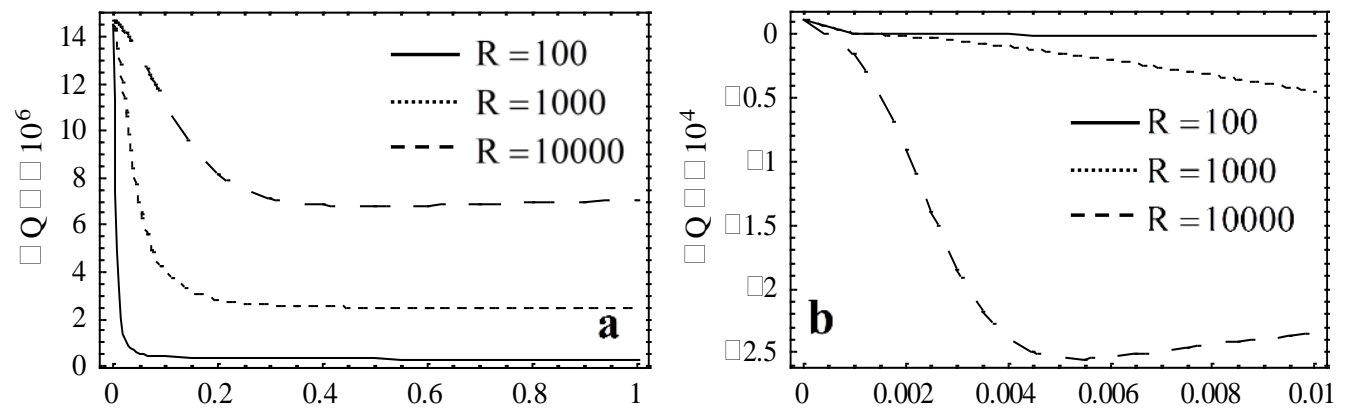

Fig. 3 Plot showing the dimensionless flow rate $\langle\mathrm{Q}\rangle$ : versus $\chi$ for $\mathrm{M}=0.002, \mathrm{k}_{\mathrm{n}}=0.01, \mathrm{~K}=0.5$, $\delta=0.2, \lambda_{1}=1000, \lambda_{2}=800, \alpha=0.001$, and $\varepsilon=0.001$ (panel a); versus $\alpha$ for $\mathrm{M}=0.001$, $\mathrm{k}_{\mathrm{n}}=0.05, \mathrm{~K}=0.05, \delta=0.001, \lambda_{1}=10000, \lambda_{2}=2000, \chi=0.6$, and $\varepsilon=0.001$ (panel b).
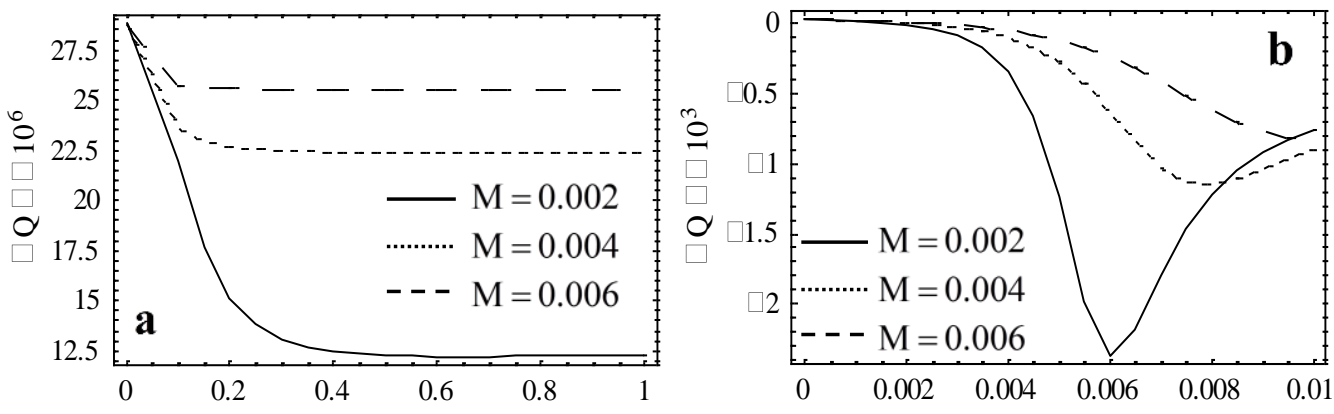

Fig. 4 Plot showing the dimensionless flow rate $\left\langle\mathrm{Q}>\right.$ : versus $\chi$ for $\mathrm{R}=10000, \mathrm{k}_{\mathrm{n}}=0.1, \mathrm{~K}=0.5$, $\delta=0.2, \lambda_{1}=1000, \lambda_{2}=1000, \alpha=0.001$, and $\varepsilon=0.001$ (panel a); versus $\alpha$ for $\mathrm{R}=10000$, $\mathrm{k}_{\mathrm{n}}=0.1, \mathrm{~K}=0.5, \delta=0.2, \lambda_{1}=1000, \lambda_{2}=800, \chi=0.6$, and $\varepsilon=0.001$ (panel b). 

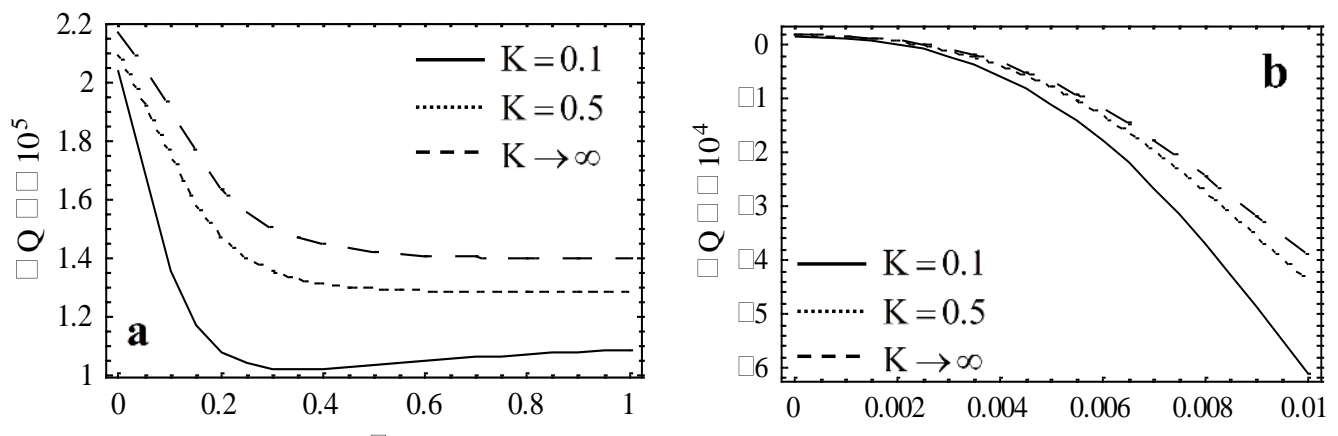

Fig. 5 Plot showing the dimensionless flow rate $\langle\mathrm{Q}\rangle$ : versus $\chi$ for $\mathrm{R}=10000, \mathrm{M}=0.002, \mathrm{k}_{\mathrm{n}}=0.1$, $\delta=0.2, \lambda_{1}=1000, \lambda_{2}=200, \alpha=0.001$, and $\varepsilon=0.001$ (panel a); versus $\alpha$ for $\mathrm{R}=10000$, $\mathrm{M}=0.004, \mathrm{k}_{\mathrm{n}}=0.05, \delta=0.2, \lambda_{1}=1000, \lambda_{2}=800, \chi=0.6$, and $\varepsilon=0.001$ (panel b).
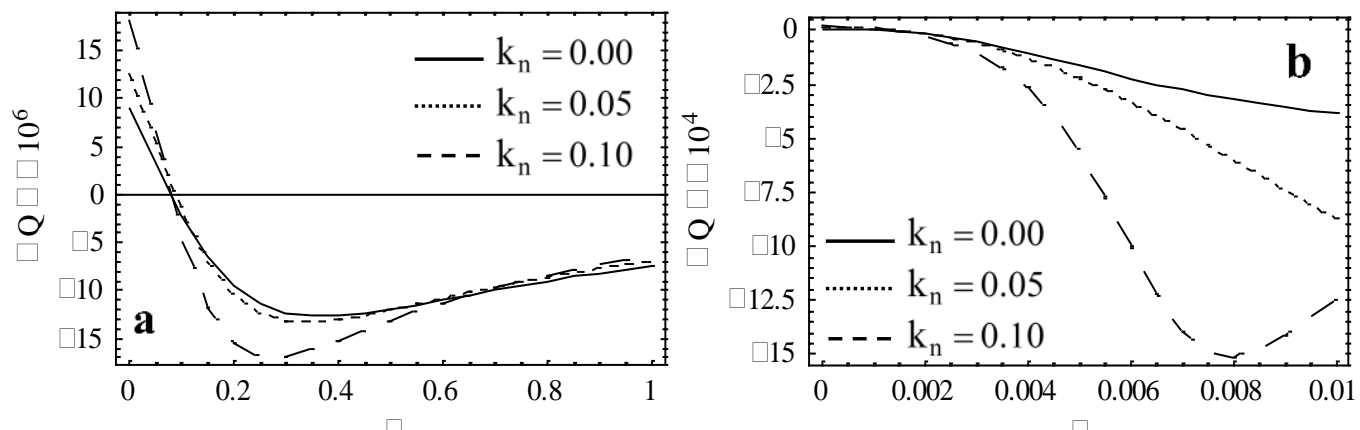

Fig. 6 Plot showing the dimensionless flow rate $\langle\mathrm{Q}>$ : versus $\chi$ for $\mathrm{R}=10000, \mathrm{M}=0.001, \mathrm{~K}=0.05$, $\delta=0.001, \lambda_{1}=1000, \lambda_{2}=400, \alpha=0.001$, and $\varepsilon=0.001$ (panel a); versus $\alpha$ for $\mathrm{R}=10000$, $\mathrm{M}=0.004, \mathrm{~K}=0.05, \delta=0.001, \lambda_{1}=1000, \lambda_{2}=800, \chi=0.6$, and $\varepsilon=0.001$ (panel b).
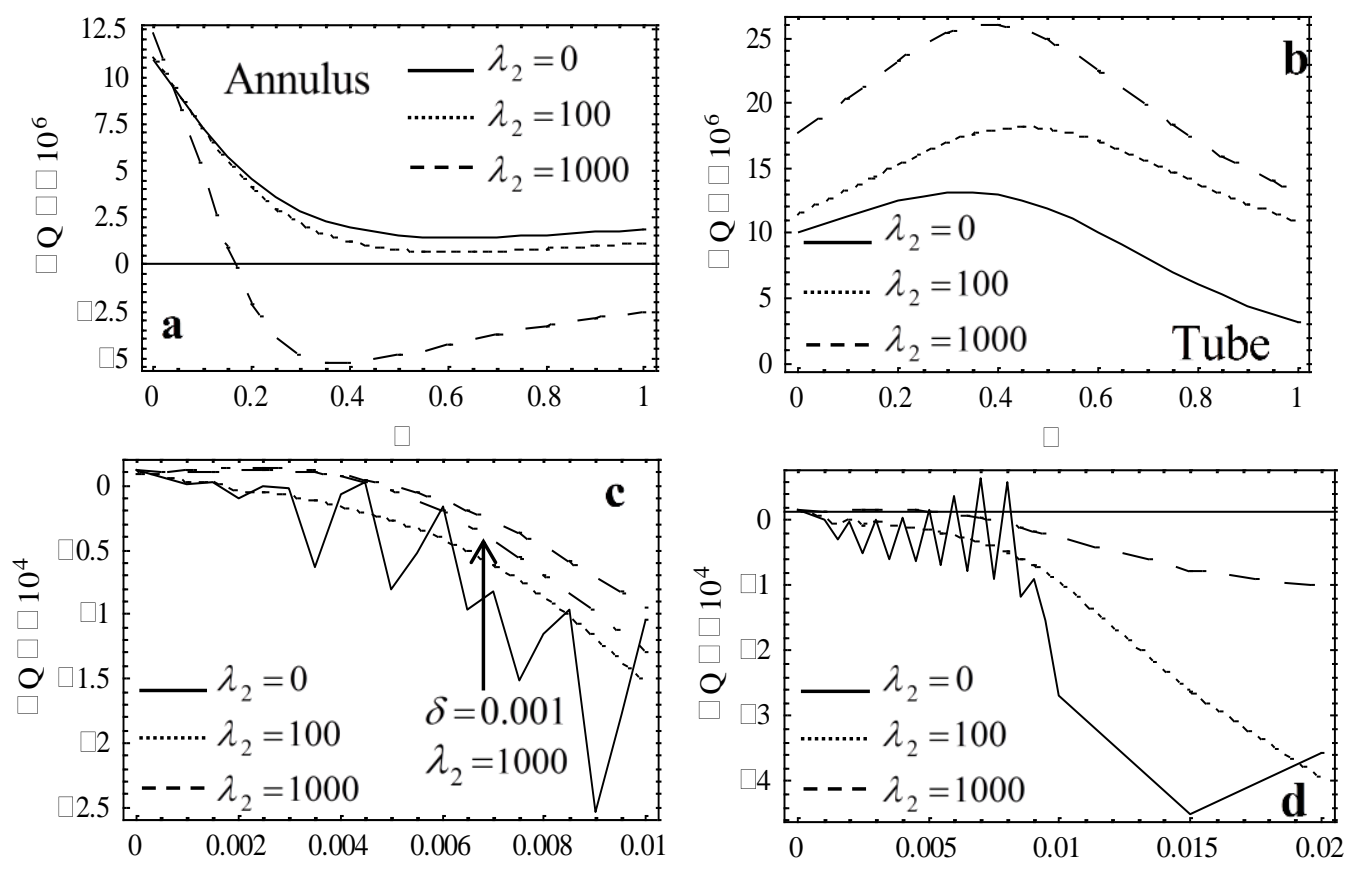

Fig. 7 Plot showing the dimensionless flow rate $\langle\mathrm{Q}\rangle$ : versus $\chi$ for $\mathrm{R}=10000, \mathrm{M}=0.001, \mathrm{k}_{\mathrm{n}}=0.01$, $\mathrm{K}=0.1, \delta=0.2, \lambda_{1}=1000, \alpha=0.001$, and $\varepsilon=0.001$ (panel a); versus $\chi$ for $\mathrm{R}=10000, \mathrm{M}=$ 
$0.001, \mathrm{k}_{\mathrm{n}}=0.01, \mathrm{~K}=0.5, \lambda_{1}=1000, \alpha=0.001$, and $\varepsilon=0.001$ (panel b); versus $\alpha$ for $\mathrm{R}=10000$, $\mathrm{M}=0.01, \mathrm{k}_{\mathrm{n}}=0.01, \mathrm{~K}=0.1, \delta=0.2, \lambda_{1}=10000, \chi=0.6$, and $\varepsilon=0.001$ (panel c); versus $\alpha$ for $\mathrm{R}=10000, \mathrm{M}=0.01, \mathrm{k}_{\mathrm{n}}=0.01, \mathrm{~K}=0.1, \delta=0.4, \lambda_{1}=10000, \chi=0.6$, and $\varepsilon=0.001$ (panel d).

\section{Conclusions}

In this study, we observed the inclusion of a magnetic field, permeability, and slip boundary effects into the flow through an annulus with peristaltic mechanism on the outer wall by using a compressible Jeffrey fluid model. The problem is solved by means of a perturbation analysis. This considered problem is important from the rheological point of view and has applications in various branches of science including stimulation of fluid flow in an annulus under the effect of elastic waves. The main findings can be summarized as follows:

1. The net flow increases with an increase in $\delta, \mathrm{M}$, and $\mathrm{K}$, while decreases with an increase in $\mathrm{R}, \mathrm{k}_{\mathrm{n}}$, and $\lambda_{2}$.

2. The backflow decreases with an increase in $\delta, \mathrm{M}, \mathrm{K}$, and $\lambda_{2}$, while increases with an increase in $\mathrm{k}_{\mathrm{n}}$.

3. It is noticed that the backflow for Newtonian fluids is more than that of nonNewtonian fluids. Also, the backflow of slip fluids is larger than that of nonslip ones.

4. The net flow rate strongly depends on $\delta$ which causes the viscoelastic behavior to become more pronounced as it increases.

5. Oscillatory decay strongly depends on $\delta$ as on $\lambda_{2}$.

6. At very low compressibility, the net flow is weakly affected by $\mathrm{M}, \mathrm{k}_{\mathrm{n}}, \mathrm{K}, \mathrm{R}$, and $\lambda_{2}$.

7. Setting $M=0, k_{n}=0, K \rightarrow \infty$, and $\lambda_{2}=0$, the results of Mekheimer and AbdelWahab [24] in an annulus are recovered.

8. The results of compressible Jeffrey fluid filling the non-porous medium in a tube of Hayat et al. [15] can be obtained by taking $\mathrm{M}=0, \mathrm{k}_{\mathrm{n}}=0, \mathrm{~K} \rightarrow \infty$, and $\delta=0$ in our investigation.

9. Our results agree with the results of Maxwell fluid of Eldesoky and Mousa [8] if we choose $\mathrm{M}=0, \mathrm{k}_{\mathrm{n}}=0, \lambda_{2}=0$, and $\delta=0$ in our investigation.

10. The results of Eldesoky [7] are recovered if we set $\mathrm{M}=0, \lambda_{2}=0$, and $\delta=0$ in our investigation.

11. Setting $\mathrm{M}=0, \mathrm{k}_{\mathrm{n}}=0, \mathrm{~K} \rightarrow \infty, \lambda_{2}=0$, and $\delta=0$ in our investigation, the results of Tsiklauri and Beresnev [39] are recovered.

12. Choosing $\mathrm{M}=0, \mathrm{k}_{\mathrm{n}}=0, \mathrm{~K} \rightarrow \infty, \lambda_{1}=\lambda_{2}=0$, and $\delta=0$ implies to a well-agreed physical situation for Newtonian fluids as obtained by Aarts and Ooms [1].

\section{References}

[1] Aarts, A. C. T., Ooms, G.: Net flow of compressible viscous liquids induced by travelling waves in porous media. J. Eng. Math. 34, 435-450 (1998).

[2] Ali, N., Hayat, T., Asghar, S.: Peristaltic flow of a Maxwell fluid in a channel with compliant walls. Chaos Solitons Fractals 39, 407-416 (2009).

[3] Barton, C., Raynor, S.: Peristaltic flow in tubes. Bull. Math. Biophysics 30, 663-680 (1968).

[4] Beavers, G. S., Joseph, D. L.: Boundary conditions at a naturally permeable wall. J. Fluid Mech. 30(1), 197-207 (1967).

[5] Bird, R. B., Curtiss, C. F., Armstrong, R. C., Hassager, O.: Dynamics of polymeric liquids, $2^{\text {nd }}$ ed. New York: Wiley 1987.

[6] Bohme, G. B., Friedrich, R.: Peristaltic flow of viscoelastic liquids. Rheol. Acta 128, 109-122 (1983).

[7] Eldesoky, I. M.: Influence of slip condition on peristaltic transport of a compressible Maxwell fluid through porous medium in a tube. Int. J. of Appl. Math and Mech. 8, 99-117 (2012). 
[8] Eldesoky, I. M., Mousa, A. A.: Peristaltic flow of a compressible non-Newtonian Maxwellian fluid through a porous medium in a tube. Int. J. Biomath. 3, 255-275 (2010).

[9] El Koumy, S. R., Barakat, E. S. B., Abdelsalam, S. I.: Hall and porous boundaries effects on peristaltic transport through porous medium of a Maxwell model. Transp. Porous Med. 94, 643-658 (2012).

[10] Elshehawey, E. F., Kaldas, S. F., Afifi, N. A.-E. S.: Peristaltic transport of a magneto- fluid through a porous medium. J. Fac. Educ., Ain Shams Univ. Egypt. 22, 35-51 (1997).

[11] Elshehawey, E. F., Sobh, A. M., Afifi, N. A. S.: Peristaltic motion of a generalized Newtonian fluid under the effect of transverse magnetic field. Proc. of the Bulgarian Academy of Sciences 53, 33-38 (2000).

[12] Elshehawy, E. F., El-Dabe, N. T., El-Desoky, I. M.: Slip effects on peristaltic flow of a nonNewtonian Maxwellian fluid. Acta Mech. 186, 141-159 (2006).

[13] Fung, Y. C., Yih, C. S.: Peristaltic transport. J.Appl. Mech. 35, 669-675 (1968).

[14] Ganiev, R. F., Ukrainskii, L. E., Frolov, K. V.: Wave mechanism for the acceleration of a liquid flowing in capillaries and porous media. Sov. Phys. Dokl. 34, 519-521 (1989).

[15] Hayat, T., Ali, N., Asghar, S.: An analysis of peristaltic transport for flow of a Jeffrey fluid. Acta Mech. 193, 101-112 (2007).

[16] Hutchison, K. J., Karpinski, E.: In vivo demonstration of flow recirculation and turbulence downstream stenoses in canine arteries. J. of Biomech. 18, 285-296 (1985).

[17] Joseph, D. D.: Fluid dynamics of viscoelastic liquids. New York: Springer 1990.

[18] Komvopoulos, K.: Surface engineering and microtribology for microelectromechanical systems. Wear 200, 305-327(1996).

[19] Ku, D. N.: Blood flow in arteries. Annu. Rev. Fluid Mech. 29, 399-434 (1997).

[20] Larson, R. G.: Constitutive equations for polymer melts and solutions. Boston London Singapore Sydney Toronto Wellington: Butterworth 1989.

[21] Mekheimer, Kh. S.: Peristaltic transport of a Newtonian fluid through a uniform and nonuniform annulus. Arab. J. Sci. Eng. 30, 69-83 (2005).

[22] Mekheimer, Kh. S., Abd Elmaboud, Y.: Peristaltic flow through a porous medium in an annulus: Application of an endoscope. Appl. Math. Inf. Sci. 2(1), 103-121 (2008).

[23] Mekheimer, Kh. S., Abd Elmaboud, Y.: Peristaltic flow of a couple stress in an annulus: Application of an endoscope. Physica A 387, 2403-2415 (2008).

[24] Mekheimer, Kh. S., Abdel-Wahab, A. N.: Compressibility effects on peristaltic flow of a nonNewtonian Maxwell fluid through an annulus. Fluid Transport: Theory, Dynamics and Applications, Nova Science Publishers, Inc. 2010.

[25] Mekheimer, Kh. S., Elkot, M. A.: Suspension model for blood flow through arterial catheterization. Chem. Eng. Commu. J. 197, 1195-1214 (2010).

[26] Mekheimer, Kh. S., El Kot, M. A.: Mathematical modeling of axial flow between two eccentric cylinders: Application on the injection of eccentric catheter through stenotic arteries. Int. J. Non-linear Mech. 47, 927-937 (2012).

[27] Nagarani, P., Sarojamma, G., Jayaraman, G.: Exact analysis of unsteady convective diffusion in Casson fluid flow in an annulus: application to catheterized artery. Acta Mech. 187, 189202 (2006).

[28] Nagarani, P., Sarojamma, G., Jayaraman, G.: Effect of boundary absorption on dispersion in Casson fluid flow in an annulus: application to catheterized artery. Acta Mech. 202, 47-63 (2009).

[29] Oldroyd, J. G.: On the formulation of the rheological equations of state. Proc. Roy. Soc. Lond. Ser. A 200, 523-541 (1950).

[30] Qi, Q., Johnson, R. E., Harris, J. G.: Boundary layer attenuation and acoustic streaming accompanying plane-wave propagation in a tube. J. Acoust. Soc. Am. 97, 1499-1509 (1995).

[31] Raju, K. K., Devanathan, R.: Peristaltic motion of non-Newtonian fluid. J. Fluid Mech. 11, 170-178 (1972).

[32] Rajagopal, K. R.: Mechanics of non-Newtonian fluids. In: Recent development in theoretical fluid mechanics, Pitman research notes in mathematics, vol. 291, pp. 129-162. New York: Longman 1993.

[33] Raptis, A., Perdikis, C., Tzivanidis, G.: Free convection flow through a porous medium bounded by a vertical surface. J. Phys. D 14, 99-102 (1981).

[34] Shapiro, A. H., Jaffrin, M. Y., Weinberg, S. L.: Peristaltic pumping with long wavelength at low Reynolds number. J. Fluid Mech. 17, 799-825 (1969).

[35] Srivastava, L. M., Srivastava, V. P.: Peristaltic transport of blood: Casson model II. J. Biomech. 17, 821-829 (1984).

[36] Srivastava, V. P.: Particle-fluid suspension flow induced by peristaltic waves in a circular cylindrical tube, Bull. Calcutta Math. Soc. 94, 167-184 (2002).

[37] Takabatake, S., Ayukawa, K., Mori, A.: Peristaltic pumping in circular cylindrical tubes: A numerical study of fluid transport and its efficiency. J. Fluid Mech. 193, 267-283 (1988). 
[38] Tan, W. C., Masuoka, T.: Stokes' first problem for an Oldroyd-B fluid in a porous half space. Phys. Fluid 17, 023101-023107 (2005).

[39] Tsiklauri, D., Beresnev, I.: Non-Newtonian effects in the peristaltic flow of a Maxwell fluid. Phys. Rev. E 64, 03630 (2001) [5 pages].

[40] Tunay, I.: Position control of catheters using magnetic fields. Mechatronics, ICM '04. Proceedings of the IEEE International Conference on, pp. 392-397, 3-5 June 2004. DOI: 10.1109/ICMECH.2004.1364471.

[41] Yin, F., Fung, Y. C.: Peristaltic waves in circular cylindrical tubes. J. Appl. Mech. 36, 579587 (1969). 